\title{
A Photochemical Entry to Depsides: Synthesis of Gustastatin
}

\section{Supporting Information}

\author{
Jorge García-Fortanet, John R. DeBergh, and Jef K. De Brabander* \\ Department of Biochemistry, The University of Texas Southwestern Medical Center at Dallas, \\ 5323 Harry Hines Boulevard, Dallas, TX 75390-9038 \\ E-mail:jdebra@biochem.swmed.edu
}

I. General Techniques: Unless otherwise noted, commercially available materials were used without further purification. All solvents were of HPLC or ACS grade. Solvents used for moisture sensitive operations were distilled from drying reagents under a nitrogen atmosphere: $\mathrm{Et}_{2} \mathrm{O}$ and THF from sodium benzophenone ketyl; benzene and toluene from sodium; $\mathrm{CH}_{2} \mathrm{Cl}_{2}$ from $\mathrm{CaH}_{2}$. Reactions were performed under an atmosphere of nitrogen with magnetic stirring unless noted otherwise. All photochemical reactions were performed with a Rayonett RPR-100 reactor fitted with a test tube carousel and $300 \mathrm{~nm}$ bulbs. Flash chromatography (FC) was performed using E Merck silicagel 60 (240-400 mesh) according to the protocol of Still, Kahn, and Mitra (J. Org. Chem. 1978, 43, 2923). Thin layer chromatography was performed using precoated plates purchased from E. Merck (silicagel 60, PF254, $0.25 \mathrm{~mm}$ ) that were visualized using a $\mathrm{KMnO}_{4}$ or Ce (IV) stain.

Nuclear magnetic resonance (NMR) spectra were recorded on a Varian Inova-400 or Mercury-300 spectrometer at operating frequencies of $400 / 300 \mathrm{MHz}\left({ }^{1} \mathrm{H} \mathrm{NMR}\right)$ or $100 / 75 \mathrm{MHz}$ $\left({ }^{13} \mathrm{C}\right.$ NMR). Chemical shifts $(\delta)$ are given in ppm relative to residual solvent (usually chloroform, $\delta 7.26$ for ${ }^{1} \mathrm{H}$ NMR or $\delta 77.23$ for proton decoupled ${ }^{13} \mathrm{C} \mathrm{NMR}$ ), and coupling constants $(J)$ in $\mathrm{Hz}$. Multiplicity is tabulated as $\mathrm{s}$ for singlet, $\mathrm{d}$ for doublet, $\mathrm{t}$ for triplet, $\mathrm{q}$ for quadruplet, and $\mathrm{m}$ for multiplet, whereby the prefix app is applied in cases where the true multiplicity is unresolved, and $b r$ when the signal in question is broadened.

Infrared spectra were recorded on a Perkin-Elmer I 1000 series FTIR with wavenumbers expressed in $\mathrm{cm}^{-1}$ using samples prepared as thin films between salt plates. UV-VIS spectra were taken in anhydrous diethyl ether and recorded with a Shimadzu UV-1601 spectrometer. Low-resolution mass spectra (LRMS) were recorded on a Shimadzu 2010-LCMS, or at the NIH regional mass spectrometry facility at the University of Washington, St. Louis, MO. Highresolution mass spectra (HRMS) were recorded at the NIH regional mass spectrometry facility at the University of Washington, St. Louis, MO. 


\section{Experimental Procedures.}

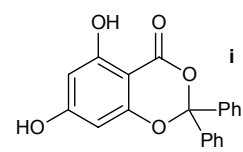

5,7-Dihydroxy-2,2-diphenyl-benzo[1,3]dioxin-4-one (i). Prepared according to the procedure described in: Dushin, R. G.; Danishefsky S. J. J. Am. Chem. Soc., 1992, 114, 655-659. To a round bottom flask containing 2,4,6trihydroxybenzoic acid monohydrate $(15 \mathrm{~g}, 71.56 \mathrm{mmol}, 1 \mathrm{eq})$ and benzophenone (39.12 g, $214.68 \mathrm{mmol}, 3 \mathrm{eq})$ was added at $0^{\circ} \mathrm{C}$ and under $\mathrm{N}_{2}$ trifluoroacetic acid $(118 \mathrm{~mL})$ and trifluoroacetic anhydride $(92 \mathrm{~mL})$. The reaction was warmed at $\mathrm{rt}$ and then stirred for $24 \mathrm{~h}$. The slightly yellow homogeneous mixture was concentrated and the residue was dissolved in toluene $(40 \mathrm{~mL})$ and concentrated again. This operation was repeated 3 times. The crude residue was purified by $\mathrm{FC}$ in a rapid fashion to minimize ketal hydrolysis (silicagel, 5\% EtOAc/Hexanes) to yield $7.6 \mathrm{~g}(32 \%)$ of i: IR (film) 3260, 1667, 1640, 1597, 1480, 1269, 1161, 1094, $695 \mathrm{~cm}^{-1} ;{ }^{1} \mathrm{H}$ NMR $\left(400 \mathrm{MHz}, \mathrm{CDCl}_{3}\right) \delta 10.20(1 \mathrm{H}, \mathrm{s}), 7.53-7.55(4 \mathrm{H}, \mathrm{m}), 7.33-7.36(6 \mathrm{H}$, $\mathrm{m}), 6.27(1 \mathrm{H}, \mathrm{s}), 6.16(1 \mathrm{H}, \mathrm{d}, J=2.0 \mathrm{~Hz}), 6.00(1 \mathrm{H}, \mathrm{d}, J=2.4 \mathrm{~Hz}) ;{ }^{13} \mathrm{C} \mathrm{NMR}\left(100 \mathrm{MHz}, \mathrm{CDCl}_{3}\right)$ $\delta 165.7,165.1,163.1,157.6,139.0,129.4,128.6,126.3,107.3,98.1,96.2$, 94.5; LRMS (ESI) Calcd for $\mathrm{C}_{20} \mathrm{H}_{13} \mathrm{O}_{5}[\mathrm{M}-\mathrm{H}]^{-}:$333.08; Found: 333.10. HRMS (ESI, added $\mathrm{NaO}_{2} \mathrm{CCF}_{3}$ ) Calcd for $\mathrm{C}_{20} \mathrm{H}_{14} \mathrm{O}_{5} \mathrm{Na}[\mathrm{MNa}]^{+}:$357.0739. Found: 357.0727 .

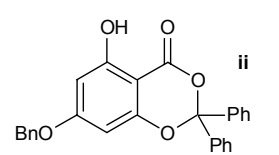

7-Benzyloxy-5-hydroxy-2,2-diphenyl-benzo[1,3]dioxin-4-one (ii). Prepared according to the procedure described in: Dushin, R. G.; Danishefsky S. J. J. Am. Chem. Soc., 1992, 114, 655-659. To a solution of diol i $(2.0 \mathrm{~g}, 5.99 \mathrm{mmol}, 1 \mathrm{eq})$ and benzyl alcohol $(651 \mu \mathrm{L}, 6.29 \mathrm{mmol}, 1.05 \mathrm{eq})$ in $\mathrm{THF}(36 \mathrm{~mL})$ at $0^{\circ} \mathrm{C}$ was added triphenylphosphine (1.66 g, $6.29 \mathrm{mmol}, 1.05 \mathrm{eq}$ ), and DIAD (1.22 mL, $6.29 \mathrm{mmol}, 1.05$ eq) and the mixture was warmed to rt over $2 \mathrm{~h}$. The solution was the diluted with EtOAc, washed trice with water and brine, dried over $\mathrm{MgSO}_{4}$, concentrated, and purified by $\mathrm{FC}$ (silicagel, 10\% EtOAc/Hexanes) to yield $1.9 \mathrm{~g}(75 \%)$ of ii: IR (film) 3209, 3066, 3035, 1695, 1644, 1583, 1503, 1353, 1268, 1103, 1026, 910, 828, 736, $699 \mathrm{~cm}^{-1} ;{ }^{1} \mathrm{H}$ NMR (400 MHz, $\left.\mathrm{CDCl}_{3}\right) \delta 10.25(1 \mathrm{H}, \mathrm{s})$, $7.54-7.56(4 \mathrm{H}, \mathrm{dd}, J=2.0 / 8.0 \mathrm{~Hz}), 7.29-7.36(11 \mathrm{H}, \mathrm{m}), 6.26(1 \mathrm{H}, \mathrm{d}, J=2 \mathrm{~Hz}), 6.12(1 \mathrm{H}, \mathrm{d}, J=$ $2.4 \mathrm{~Hz}), 4.99(2 \mathrm{H}, \mathrm{s}) ;{ }^{13} \mathrm{C}$ NMR $\left(100 \mathrm{MHz}, \mathrm{CDCl}_{3}\right) \delta 166.7,165.1,163.1,157.1,139.1,135.4$, 129.3, 128.6, 128.5, 128.3, 127.5, 126.3, 107.2, 96.7, 95.8, 94.8, 70.4; LRMS (ESI) Calcd for $\mathrm{C}_{27} \mathrm{H}_{19} \mathrm{O}_{5}[\mathrm{M}-\mathrm{H}]^{-}:$423.12; Found: 423.25. HRMS (ESI, added $\mathrm{NaO}_{2} \mathrm{CCF}_{3}$ ) Calcd for $\mathrm{C}_{27} \mathrm{H}_{20} \mathrm{O}_{5} \mathrm{Na}[\mathrm{MNa}]^{+}$: 447.1208. Found: 447.1230.

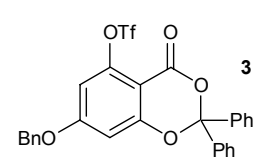

Trifluoro-methanesulfonic

acid 7-benzyloxy-4-oxo-2,2-diphenyl-4Hbenzo[1,3]dioxin-5-yl ester (3). To a solution of phenol ii (1.9 g, $4.48 \mathrm{mmol}, 1$ eq) and pyridine $(1.81 \mathrm{~mL}, 22.40 \mathrm{mmol}, 5 \mathrm{eq})$ in $\mathrm{CH}_{2} \mathrm{Cl}_{2}(33 \mathrm{~mL})$ was added trifluoromethanesulfonic anhydride $(980 \mu \mathrm{L}, 5.83 \mathrm{mmol}, 1.3 \mathrm{eq})$ dropwise at $0^{\circ} \mathrm{C}$. After stirring for $1.5 \mathrm{~h}$ at $\mathrm{rt}$ and under $\mathrm{N}_{2}$, the reaction was transferred into a separation funnel containing sat. $\mathrm{NaHCO}_{3}$, the organic phase was separated and the aqueous phase was washed with $\mathrm{CH}_{2} \mathrm{Cl}_{2}(3 \times 50 \mathrm{~mL})$. The combined organic phases were washed with water and brine and dried over $\mathrm{MgSO}_{4}$. After concentration and purification by FC (silicagel, 20\% EtOAc/Hexanes), 4.2 g (94\%) of 3 was obtained: IR (film) 3066, 2360, 1746, 1627, 1575, 1431, 1272, 1210, 1153, 1058, 1029, 816, $698 \mathrm{~cm}^{-1} ;{ }^{1} \mathrm{H}$ NMR (400 MHz, $\left.\mathrm{CDCl}_{3}\right) \delta 7.52-7.55$ (4H, m), $7.32-7.42(11 \mathrm{H}, \mathrm{m}), 6.73(1 \mathrm{H}, \mathrm{d}, J=2.4 \mathrm{~Hz}), 6.54(1 \mathrm{H}, \mathrm{d}, J=2.4 \mathrm{~Hz}), 5.10(2 \mathrm{H}, \mathrm{s}) ;{ }^{13} \mathrm{C}$ NMR $\left(100 \mathrm{MHz}, \mathrm{CDCl}_{3}\right) \delta 164.6,159.1,156.9,150.0,138.7,134.5,129.4,128.8,128.7,128.6,127.7$, 126.4, 107.2, 106.3, 102.4, 71.2; LRMS (ESI) Calcd for $\mathrm{C}_{28} \mathrm{H}_{19} \mathrm{~F}_{3} \mathrm{NaO}_{7} \mathrm{~S}[\mathrm{M}+\mathrm{Na}]^{+}$: 579.07; 
Found: 579.00. HRMS (ESI, added $\mathrm{NaO}_{2} \mathrm{CCF}_{3}$ ) Calcd for $\mathrm{C}_{28} \mathrm{H}_{19} \mathrm{O}_{7} \mathrm{~F}_{3} \mathrm{SNa}$ [MNa] $]^{+}$579.0701. Found: 579.0698.

$\mathrm{H}_{4} \mathrm{BF}_{3} \mathrm{~K}$ Potassium trans-1-Hep-1-enyl Trifluoroborate (7). Prepared according to the procedure described in: Molander, G.A.; Bernardi, C. R. J. Org. Chem. 2002, 67, 8424-8429. To a solution of 1-heptyne (1.34 mL, $10 \mathrm{mmol}, 1 \mathrm{eq})$ in $\mathrm{CH}_{2} \mathrm{Cl}_{2}(5 \mathrm{~mL})$ at $0^{\circ} \mathrm{C}$ and under $\mathrm{N}_{2}$ was added $\mathrm{Br}_{2} \mathrm{BH} \cdot \mathrm{SMe}_{2}\left(1 \mathrm{M}\right.$ in $\left.\mathrm{CH}_{2} \mathrm{Cl}_{2}, 10 \mathrm{mmol}, 10 \mathrm{~mL}, 1 \mathrm{eq}\right)$. After stirring at rt for $4 \mathrm{~h}$, the light yellow solution was added via syringe to a mixture of diethyl ether/water $(10 \mathrm{~mL}$ of $\mathrm{Et}_{2} \mathrm{O} / 4 \mathrm{~mL}$ of $\mathrm{H}_{2} \mathrm{O}$ ) at $0^{\circ} \mathrm{C}$. The mixture was stirred at $\mathrm{rt}$ for $15 \mathrm{~min}$. The aqueous layer was separated, and the organic phase was washed with cold water and then brine. The organic phase was subsequently dried over $\mathrm{MgSO}_{4}$ and concentrated to provide $1.05 \mathrm{~g}$ (74\%) of trans-1-hep-1enyl boronic acid as a white solid.

To a solution of the above prepared trans-1-hep-1-enyl boronic acid (1.05 g, $7.41 \mathrm{mmol}, 1 \mathrm{eq})$ in $\mathrm{Et}_{2} \mathrm{O}(15 \mathrm{~mL})$ was added $\mathrm{KHF}_{2}(1.62 \mathrm{~g}, 20.75 \mathrm{mmol}, 2.8 \mathrm{eq})$ followed by $\mathrm{H}_{2} \mathrm{O}(6.7 \mathrm{~mL})$ over 30 min. After stirring at $\mathrm{rt}$ for $3 \mathrm{~h}$, the solution was concentrated, and the crude material was dissolved in acetone, filtered, and concentrated. The resulting white solid was purified by dissolving in hot acetone and precipitating with $\mathrm{Et}_{2} \mathrm{O}$ to afford $1.26 \mathrm{~g}(83 \%)$ of 7 as a white solid: IR (film) 2959, 2926, 1647, 1111, $933 \mathrm{~cm}^{-1}$; ${ }^{1} \mathrm{H}$ NMR (400 MHz, DMSO-d 6 ) $\delta 5.49(1 \mathrm{H}, \mathrm{dt}, J=$ $6.1 / 17.1 \mathrm{~Hz}), 5.20-5.27(1 \mathrm{H}, \mathrm{dm}, J=17.6 \mathrm{~Hz}), 1.88(2 \mathrm{H}, \mathrm{q}, J=6.0 \mathrm{~Hz}), 1.21-1.32(6 \mathrm{H}, \mathrm{m})$, $0.87(3 \mathrm{H}, \mathrm{t}, J=7.2 \mathrm{~Hz}) ;{ }^{13} \mathrm{C}$ NMR $\left(75 \mathrm{MHz}, \mathrm{DMSO}-d_{6}\right) \delta 133.7,133.65,35.4,31.1,28.9,22.1$, 14.0 .

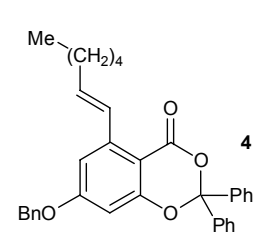

7-Benzyloxy-5-hept-1-enyl-2,2-diphenyl-benzo[1,3]dioxin-4-one

(4). Prepared according to the procedure described in: Molander, G.A.; Bernardi, C. R. J. Org. Chem. 2002, 67, 8424-8429. A solution of trifluoroborate 7 (102 mg, $0.5 \mathrm{mmol}, 1 \mathrm{eq}), \mathrm{PdCl}_{2}$ (dppf) $\mathrm{CH}_{2} \mathrm{Cl}_{2}(8.1 \mathrm{mg}, 0.001 \mathrm{mmol}, 2 \mathrm{~mol} \%)$, triflate 3 (278 mg, $0.5 \mathrm{mmol}, 1 \mathrm{eq})$ in ${ }^{i} \mathrm{PrOH} / \mathrm{H}_{2} \mathrm{O}(2: 1,5 \mathrm{~mL}$, degassed) was added at $\mathrm{rt}$ and under $\mathrm{N}_{2}{ }^{t} \mathrm{BuNH}_{2}(158 \mu \mathrm{L}, 1.5 \mathrm{mmol}, 3 \mathrm{eq})$. After refluxing overnight, the reaction mixture was cooled to $\mathrm{rt}$ and diluted with water $(10 \mathrm{~mL})$, followed by extraction of diethyl ether. The organic layers were combined and washed with brine, dried over $\mathrm{MgSO}_{4}$, and concentrated. After purification by FC (silicagel, 10\% EtOAc/Hexanes), $201 \mathrm{mg}(80 \%)$ of pure 4 was obtained: IR (film) 3065, 3034, 2927, 2857, 1731, 1605, 1570, 1452, 1452, 1266, 1208, 1160, 970, 697 $\mathrm{cm}^{-1}$; ${ }^{1} \mathrm{H}$ NMR $\left(400 \mathrm{MHz}, \mathrm{CDCl}_{3}\right) \delta 7.52-7.55(4 \mathrm{H}, \mathrm{dd}, J=1.2 / 7.6 \mathrm{~Hz}), 7.25-7.39(11 \mathrm{H}, \mathrm{m})$, $6.74(1 \mathrm{H}, \mathrm{d}, J=2.4 \mathrm{~Hz}), 6.57(1 \mathrm{H}, \mathrm{d}, J=2.0 \mathrm{~Hz}), 6.11(1 \mathrm{H}, \mathrm{dt}, J=6.8 / 16.0 \mathrm{~Hz}), 5.07(2 \mathrm{H}, \mathrm{s})$, $2.20(2 \mathrm{H}, \mathrm{q}, J=7.2 \mathrm{~Hz}), 1.44(2 \mathrm{H}$, app pent, $J=7.2 \mathrm{~Hz}), 1.26-1.32(4 \mathrm{H}, \mathrm{m}), 0.88(3 \mathrm{H}, \mathrm{t}, J=6.8$ $\mathrm{Hz}) ;{ }^{13} \mathrm{C}$ NMR $\left(100 \mathrm{MHz}, \mathrm{CDCl}_{3}\right) \delta 163.8,160.0,158.9,144.5,139.8,135.7,135.6,129.0$, 128.7, 128.4, 128.3, 127.9, 127.5, 126.4, 108.9, 105.6, 105.2, 101.1, 70.3, 33.0, 31.4, 28.7, 22.4, 14.0; LRMS (ESI) Calcd for $\mathrm{C}_{34} \mathrm{H}_{32} \mathrm{NaO}_{4}[\mathrm{MNa}]^{+}$: 527.22; Found: 527.20. HRMS (ESI, added $\mathrm{NaO}_{2} \mathrm{CCF}_{3}$ ) Calcd for $\mathrm{C}_{34} \mathrm{H}_{32} \mathrm{O}_{4} \mathrm{Na}[\mathrm{MNa}]^{+}: 527.2198$. Found: 527.2212.

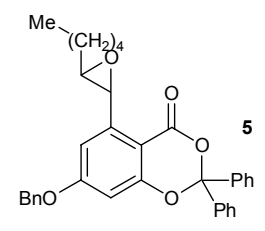

7-Benzyloxy-5-(3-pentyl-oxiranyl)-2,2-diphenyl-benzo[1,3]dioxin-4-one (5). To a solution of alkene 4 (198 mg, $0.393 \mathrm{mmol}, 1$ eq) in $\mathrm{CH}_{2} \mathrm{Cl}_{2} / \mathrm{pH} 7$ buffer $(1: 1,8 \mathrm{~mL})$ was added at $0^{\circ} \mathrm{C} m$-CPBA $(268 \mathrm{mg}, \sim 1.12 \mathrm{mmol}, \sim 2.85 \mathrm{eq})$. After stirring at $\mathrm{rt}$ for $3 \mathrm{~h}$, the organic phase was separated and the aqueous phase was washed with $\mathrm{Et}_{2} \mathrm{O}(2 \times 50 \mathrm{~mL})$. The combined organic phases were washed with 
sat. $\mathrm{Na}_{2} \mathrm{~S}_{2} \mathrm{O}_{3}$, and brine, dried over $\mathrm{MgSO}_{4}$, and concentrated. Purification of the residue by FC (silicagel, 10\% EtOAc/Hexanes) afforded $162 \mathrm{mg}$ (79\%) of 5: IR (film) 3065, 2956, 2930, 1731, 1613, 1580, 1453, 1442, 1270, 1160, $698 \mathrm{~cm}^{-1} ;{ }^{1} \mathrm{H}$ NMR (400 MHz, $\left.\mathrm{CDCl}_{3}\right) \delta 7.54-7.58(4 \mathrm{H}$, $\mathrm{m}), 7.28-7.38(11 \mathrm{H}, \mathrm{m}), 6.72(1 \mathrm{H}, \mathrm{d}, J=2.4 \mathrm{~Hz}), 6.61(1 \mathrm{H}, \mathrm{d}, J=2.4 \mathrm{~Hz}), 5.06(2 \mathrm{H}, \mathrm{s}), 4.32$ $(1 \mathrm{H}, \mathrm{d}, J=1.6 \mathrm{~Hz}), 2.64(1 \mathrm{H}, \mathrm{td}, J=2.0 / 5.6 \mathrm{~Hz}), 1.64-1.81(2 \mathrm{H}, \mathrm{m}), 1.54(2 \mathrm{H}, \mathrm{m}), 1.34(4 \mathrm{H}$, $\mathrm{m}), 0.90(3 \mathrm{H}, \mathrm{t}, J=7.2 \mathrm{~Hz}) ;{ }^{13} \mathrm{C} \mathrm{NMR}\left(100 \mathrm{MHz}, \mathrm{CDCl}_{3}\right) \delta 164.9,160.0,158.8,145.4,139.8$, $139.5,135.5,129.1,129.1,128.6,128.5,128.3,127.5,126.5,126.2,106.8,106.2,106.0,102.0$, 70.3, 63.4, 57.0, 32.2, 31.5, 25.3, 22.5, 14.0; LRMS (ESI) Calcd for $\mathrm{C}_{34} \mathrm{H}_{32} \mathrm{NaO}_{5}[\mathrm{MNa}]^{+}$: 543.21; Found: 543.15. HRMS (ESI, added $\mathrm{NaO}_{2} \mathrm{CCF}_{3}$ ) Calcd for $\mathrm{C}_{34} \mathrm{H}_{32} \mathrm{O}_{5} \mathrm{Na}[\mathrm{MNa}]^{+}$: 543.2147. Found: 543.2153.

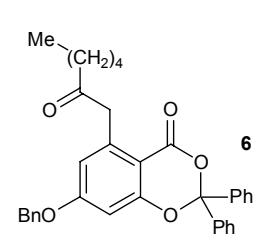

7-Benzyloxy-5-(2-oxo-heptyl)-2,2-diphenyl-benzo[1,3]dioxin-4-one (6). To a solution of epoxide 5 (440 $\mathrm{mg}, 0.85 \mathrm{mmol}, 1 \mathrm{eq})$, and $\mathrm{Pd}(\mathrm{OAc})_{2}(20 \mathrm{mg}, 0.09$ mmol, $10 \% \mathrm{~mol}$ ) in ${ }^{t} \mathrm{BuOH}$, (degassed, $20 \mathrm{~mL}$ ) was added at $\mathrm{rt}$ and under $\mathrm{N}_{2}$, $\mathrm{PBu}_{3}(64 \mu \mathrm{L}, 0.255 \mathrm{mmol}, 30 \mathrm{~mol} \%)$. The reaction mixture was stirred at reflux for $4 \mathrm{~h}$. (TLC monitoring). When the starting material was consumed, the solvent was removed under reduced pressure and the residue purified by FC (silicagel, 15\% EtOAc/Hexanes) to afford $370 \mathrm{mg}$ (84\%) of pure 6: IR (film) 3064, 2955, 2930, 1729, 1614, 1578, 1441, 1273, 1162, 1074, 734, $700 \mathrm{~cm}^{-1} ;{ }^{1} \mathrm{H}$ NMR $\left(400 \mathrm{MHz}, \mathrm{CDCl}_{3}\right) \delta$ $7.52-7.55(4 \mathrm{H}, \mathrm{dd}, J=2.0 / 8.0 \mathrm{~Hz}), 7.28-7.38(11 \mathrm{H}, \mathrm{m}), 6.62(1 \mathrm{H}, \mathrm{d}, J=2.4 \mathrm{~Hz}), 6.41(1 \mathrm{H}, \mathrm{d}, J$ $=2.0 \mathrm{~Hz}), 5.04(2 \mathrm{H}, \mathrm{s}), 3.94(2 \mathrm{H}, \mathrm{s}), 2.46(2 \mathrm{H}, \mathrm{t}, J=7.4 \mathrm{~Hz}), 1.56(2 \mathrm{H}$, app pent, $J=7.6 \mathrm{~Hz})$, $1.21-1.32(4 \mathrm{H}, \mathrm{m}), 0.88(3 \mathrm{H}, \mathrm{t}, J=7.2 \mathrm{~Hz}) ;{ }^{13} \mathrm{C} \mathrm{NMR}\left(100 \mathrm{MHz}, \mathrm{CDCl}_{3}\right) \delta 206.7,164.0,160.4$, 159.2, 141.2, 139.6, 135.4, 129.0, 128.6, 128.4, 128.3, 127.5, 126.4, 115.0, 106.6, 106.0, 101.6, 70.3, 48.4, 42.4, 31.3, 23.1, 22.4, 13.9; LRMS (ESI) Calcd for $\mathrm{C}_{34} \mathrm{H}_{32} \mathrm{NaO}_{5}[\mathrm{MNa}]^{+}: 543.21$; Found: 543.21. HRMS (ESI, added $\mathrm{NaO}_{2} \mathrm{CCF}_{3}$ ) Calcd for $\mathrm{C}_{34} \mathrm{H}_{32} \mathrm{O}_{5} \mathrm{Na}$ [MNa] ${ }^{+}: 543.2147$. Found: 543.2159 .

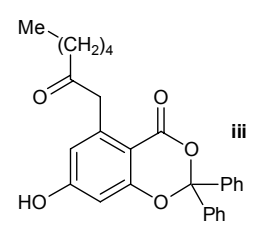

7-Hydroxy-5-(2-oxo-heptyl)-2,2-diphenyl-benzo[1,3]dioxin-4-one (iii). To a suspension of catalyst (10\% Pd on carbon, $20 \mathrm{mg}, 0.02 \mathrm{mmol}, 10 \mathrm{~mol} \%)$ in EtOAc $(1 \mathrm{~mL})$ was added at $\mathrm{rt}$ and under $\mathrm{H}_{2}$ ketone $6(100 \mathrm{mg}, 0.192 \mathrm{mmol}, 1$ eq) in EtOAc $(2 \mathrm{~mL})$. After stirring under $\mathrm{H}_{2}$ (balloon pressure) at $\mathrm{rt}$ for $5 \mathrm{~h}$ (TLC monitoring), the reaction mixture was filtered through a pad of celite and washed with EtOAc. The filtrate was concentrated and purified by FC (silicagel, 50\% EtOAc/Hexanes) to afford $74 \mathrm{mg}$ (90\%) of compound iii: IR (film) 3282, 2956, 2931, 1726, $1615,1451,1274,1208,1164,1074,911,733,701 \mathrm{~cm}^{-1} ;{ }^{1} \mathrm{H} \mathrm{NMR}\left(400 \mathrm{MHz}, \mathrm{CDCl}_{3}\right) \delta 8.30$ (1H, br), 7.49-7.51 (4H, dd, $J=1.6 / 8.0 \mathrm{~Hz}), 7.21-7.29(6 \mathrm{H}, \mathrm{m}), 6.36(1 \mathrm{H}, \mathrm{d}, J=2.4 \mathrm{~Hz}), 6.08$ $(1 \mathrm{H}, \mathrm{d}, J=2.0 \mathrm{~Hz}), 3.85(2 \mathrm{H}, \mathrm{s}), 2.57(2 \mathrm{H}, \mathrm{t}, J=7.4 \mathrm{~Hz}), 1.58(2 \mathrm{H}$, app pent, $J=7.2 \mathrm{~Hz})$, $1.22-1.33(4 \mathrm{H}, \mathrm{m}), 0.87(3 \mathrm{H}, \mathrm{t}, J=7.0 \mathrm{~Hz}) ;{ }^{13} \mathrm{C} \mathrm{NMR}\left(100 \mathrm{MHz}, \mathrm{CDCl}_{3}\right) \delta 211.0,163.4,161.3$, 159.4, 140.2, 139.6, 129.0, 128.4, 126.3, 116.2, 106.0, 105.2, 103.3, 48.5, 42.7, 31.2, 23.1, 22.4, 13.9; LRMS (ESI) Calcd for $\mathrm{C}_{27} \mathrm{H}_{26} \mathrm{NaO}_{5}[\mathrm{MNa}]^{+}$: 453.17; Found: 453.10. HRMS (ESI, added $\mathrm{NaO}_{2} \mathrm{CCF}_{3}$ ) Calcd for $\mathrm{C}_{27} \mathrm{H}_{26} \mathrm{O}_{5} \mathrm{Na}[\mathrm{MNa}]^{+}: 453.1678$. Found: 453.1659 .

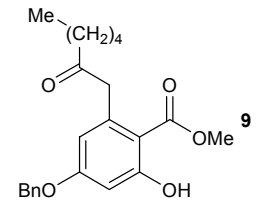

4-Benzyloxy-2-hydroxy-6-(2-oxo-heptyl)-benzoic acid methyl ester (9). To an ovendried borosilicate test tube sealed with a rubber septum was added ketone 6 (100 mg, $0.193 \mathrm{mmol}$, 1eq) in freshly distilled THF $(0.1 \mathrm{M}, 1.9 \mathrm{~mL})$ followed by addition of $\mathrm{MeOH}(24 \mu \mathrm{L}, 0.577 \mathrm{mmol}, 3$ eq). This reaction 
solution was then placed in a Rayonett reactor and photolyzed for $5 \mathrm{~h}$ at $300 \mathrm{~nm}$. The solution was then concentrated and purified by FC (silicagel, 5\% EtOAc/Hexanes) to afford $63 \mathrm{mg}(89 \%)$ of pure ester 9: IR (film) 2954, 2930, 1719, 1658, 1619, 1579, 1438, 1331, 1258, 1166, 1029, $737,699 \mathrm{~cm}^{-1}$; ${ }^{1} \mathrm{H}$ NMR $\left(400 \mathrm{MHz}, \mathrm{CDCl}_{3}\right) \delta 11.60(1 \mathrm{H}, \mathrm{s}), 7.32-7.41(5 \mathrm{H}, \mathrm{m}), 6.49(1 \mathrm{H}, \mathrm{d}, J=$ $2.4 \mathrm{~Hz}), 6.32(1 \mathrm{H}, \mathrm{d}, J=2.4 \mathrm{~Hz}), 5.05(2 \mathrm{H}, \mathrm{s}), 3.89(2 \mathrm{H}, \mathrm{s}), 3.82(3 \mathrm{H}, \mathrm{s}), 2.41(2 \mathrm{H}, \mathrm{t}, J=7.4 \mathrm{~Hz})$, $1.58(2 \mathrm{H}$, app pent, $J=7.2 \mathrm{~Hz}), 1.24-1.34(4 \mathrm{H}, \mathrm{m}), 0.89(3 \mathrm{H}, \mathrm{t}, J=7.0 \mathrm{~Hz}) ;{ }^{13} \mathrm{C}$ NMR $(100$ $\left.\mathrm{MHz}, \mathrm{CDCl}_{3}\right) \delta 207.4,170.8,165.6,163.2,138.9,135.9,128.6,128.2,127.5,113.4,105.2$, 100.9, 70.0, 51.8, 51.2, 41.9, 31.4, 23.3, 22.4, 13.9; LRMS (ESI) Calcd for $\mathrm{C}_{22} \mathrm{H}_{26} \mathrm{NaO}_{5}[\mathrm{MNa}]^{+}$: 393.17; Found: 393.10. HRMS (ESI, added $\mathrm{NaO}_{2} \mathrm{CCF}_{3}$ ) Calcd for $\mathrm{C}_{22} \mathrm{H}_{26} \mathrm{O}_{5} \mathrm{Na}[\mathrm{MNa}]^{+}$: 393.1678. Found: 393.1677.

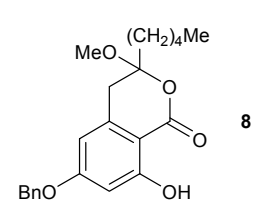

6-Benzyloxy-8-hydroxy-3-methoxy-3-pentyl-isochroman-1-one (8). ${ }^{1} \mathrm{H}$ NMR $\left(300 \mathrm{MHz}, \mathrm{CDCl}_{3}\right) \delta 11.20(1 \mathrm{H}, \mathrm{d}, J=0.6 \mathrm{~Hz}), 7.37-7.40(5 \mathrm{H}, \mathrm{m}), 6.43(1 \mathrm{H}, \mathrm{d}$, $J=2.4 \mathrm{~Hz}), 6.34(1 \mathrm{H}, \mathrm{d}, J=2.1 \mathrm{~Hz}), 6.06(2 \mathrm{H}, \mathrm{s}), 3.34(3 \mathrm{H}, \mathrm{s}), 3.10(1 \mathrm{H}$, app d, $J=16.2 \mathrm{~Hz}), 3.04(1 \mathrm{H}$, app d, $J=16.5 \mathrm{~Hz}), 2.00(1 \mathrm{H}, \mathrm{m}), 1.88(1 \mathrm{H}, \mathrm{m})$, $1.35-1.45(6 \mathrm{H}, \mathrm{m}), 0.92(3 \mathrm{H}, \mathrm{t}, J=6.5 \mathrm{~Hz}) ;{ }^{13} \mathrm{C} \mathrm{NMR}\left(75 \mathrm{MHz}, \mathrm{CDCl}_{3}\right) \delta$ $168.6,165.0,164.3,144.2,139.5,135.8,128.7,128.6,128.3,127.5,127.2$, 126.9, 107.6, 107.0, $100.2,70.2,49.8,36.2,35.0,31.7,23.1,22.5,13.9$.

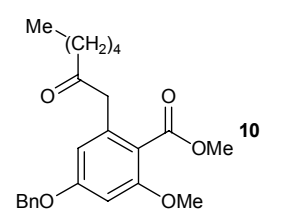

4-Benzyloxy-2-methoxy-6-(2-oxo-heptyl)-benzoic acid methyl ester (10). A solution of phenol 9 (200 mg, $0.541 \mathrm{mmol}, 1 \mathrm{eq}), \mathrm{Me}_{3} \mathrm{OBF}_{4}(400 \mathrm{mg}, 2.70$ mmol, $5 \mathrm{eq}$ ), and proton-sponge (694 mg, $3.25 \mathrm{mmol}, 6 \mathrm{eq})$ in $\mathrm{CH}_{2} \mathrm{Cl}_{2}(12 \mathrm{~mL})$ was stirred at $\mathrm{rt}$ and under $\mathrm{N}_{2}$ for $48 \mathrm{~h}$, after which the reaction was diluted with EtOAc $(20 \mathrm{~mL})$, filtered through a pad of celite and washed with water $(2 \times$ $50 \mathrm{~mL})$ and aqueous $\mathrm{CuSO}_{4}(1 \mathrm{M}, 50 \mathrm{~mL})$. The organic phase was dried over $\mathrm{MgSO}_{4}$, concentrated and purified by FC (silicagel, 20\% EtOAc/Hexanes) to afford $169 \mathrm{mg}(81 \%)$ of pure 10: IR (film) 2952, 1720, 1604, 1456, 1430, 1275, 1161, 1098, 1039, 739, $699 \mathrm{~cm}^{-1} ;{ }^{1} \mathrm{H}$ NMR $\left(400 \mathrm{MHz}, \mathrm{CDCl}_{3}\right) \delta 7.32-7.42(5 \mathrm{H}, \mathrm{m}), 6.48(1 \mathrm{H}, \mathrm{d}, J=2.4 \mathrm{~Hz}), 6.39(1 \mathrm{H}, \mathrm{d}, J=2.0 \mathrm{~Hz})$, $5.06(2 \mathrm{H}, \mathrm{s}), 3.84(3 \mathrm{H}, \mathrm{s}), 3.79(3 \mathrm{H}, \mathrm{s}), 3.66(2 \mathrm{H}, \mathrm{s}), 2.40(2 \mathrm{H}, \mathrm{t}, J=7.4 \mathrm{~Hz}), 1.54(2 \mathrm{H}$, app pent, $J=7.4 \mathrm{~Hz}), 1.20-1.31(4 \mathrm{H}, \mathrm{m}), 0.87(3 \mathrm{H}, \mathrm{t}, J=7.0 \mathrm{~Hz}) ;{ }^{13} \mathrm{C} \mathrm{NMR}\left(100 \mathrm{MHz}, \mathrm{CDCl}_{3}\right) \delta 207.5$, 168.0, 160.8, 158.9, 136.2, 135.7, 128.6, 128.2, 127.5, 116.3, 108.2, 98.5, 70.1, 56.0, 52.0, 48.2, 41.8, 31.3, 23.3, 22.4, 13.9; LRMS (ESI) Calcd for $\mathrm{C}_{23} \mathrm{H}_{28} \mathrm{NaO}_{5}$ [MNa] ${ }^{+}$: 407.18; Found: 407.10. HRMS (ESI, added $\mathrm{NaO}_{2} \mathrm{CCF}_{3}$ ) Calcd for $\mathrm{C}_{23} \mathrm{H}_{28} \mathrm{O}_{5} \mathrm{~K}[\mathrm{MK}]^{+}:$423.1574. Found: 423.1584 .

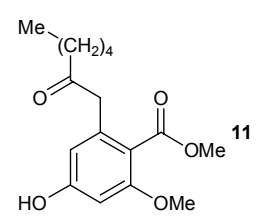

4-Hydroxy-2-methoxy-6-(2-oxo-heptyl)-benzoic acid methyl ester (11). To a suspension of catalyst (10\% Pd on carbon, $20 \mathrm{mg}, 0.02 \mathrm{mmol})$ in EtOAc $(0.5$ $\mathrm{mL}$ ) was added at $\mathrm{rt}$ and under $\mathrm{H}_{2}$ ketone 10 (23.7 $\left.\mathrm{mg}, 0.0617 \mathrm{mmol}, 1 \mathrm{eq}\right)$ in EtOAc $(1 \mathrm{~mL})$. After stirring under $\mathrm{H}_{2}$ (balloon pressure) at $\mathrm{rt}$ for $3 \mathrm{~h}$ (TLC monitoring), the reaction mixture was filtered through a pad of celite and washed with EtOAc. The filtrate was concentrated and purified by FC (silicagel, 50\% EtOAc/Hexanes) to afford $14.6 \mathrm{mg}(80 \%)$ of compound pure phenol 11: IR (film) 3365, 2955, $1713,1609,1593,1469,1434,1277,1194,1165,1099,836 \mathrm{~cm}^{-1} ;{ }^{1} \mathrm{H} \mathrm{NMR}\left(400 \mathrm{MHz}, \mathrm{CDCl}_{3}\right) \delta$ $7.02(1 \mathrm{H}, \mathrm{br}), 6.28(1 \mathrm{H}, \mathrm{d}, J=2.0 \mathrm{~Hz}), 6.22(1 \mathrm{H}, \mathrm{d}, J=2.0 \mathrm{~Hz}), 3.83(3 \mathrm{H}, \mathrm{s}), 3.72(3 \mathrm{H}, \mathrm{s}), 3.65$ $(2 \mathrm{H}, \mathrm{s}), 2.47(2 \mathrm{H}, \mathrm{t}, J=7.4 \mathrm{~Hz}), 1.56(2 \mathrm{H}$, app pent, $J=7.2 \mathrm{~Hz}), 1.20-1.32(4 \mathrm{H}, \mathrm{m}), 0.87(3 \mathrm{H}, \mathrm{t}$, $J=7.0 \mathrm{~Hz}) ;{ }^{13} \mathrm{C} \mathrm{NMR}\left(100 \mathrm{MHz}, \mathrm{CDCl}_{3}\right) \delta 209.6,168.3,159.3,158.9,135.5,114.8,110.2$, 98.4, 55.8, 52.1, 48.0, 42.1, 31.2, 23.3, 22.4, 13.9; LRMS (ESI) Calcd for $\mathrm{C}_{16} \mathrm{H}_{22} \mathrm{NaO}_{5}[\mathrm{MNa}]^{+}$: 
317.14; Found: 317.05. HRMS (ESI, added $\mathrm{NaO}_{2} \mathrm{CCF}_{3}$ ) Calcd for $\mathrm{C}_{16} \mathrm{H}_{22} \mathrm{O}_{5} \mathrm{Na}$ [MNa] $]^{+}$: 317.1365. Found: 317.1354 .

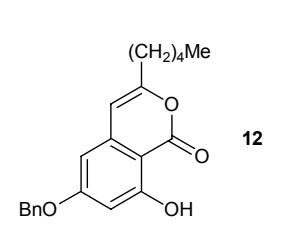

6-Benzyloxy-8-hydroxy-3-pentyl-isochromen-1-one (12). ${ }^{1} \mathrm{H} \quad \mathrm{NMR} \quad(400$ $\left.\mathrm{MHz}, \mathrm{CDCl}_{3}\right) \delta 11.12(1 \mathrm{H}, \mathrm{s}), 7.35-7.42(5 \mathrm{H}, \mathrm{m}), 6.53(1 \mathrm{H}, \mathrm{d}, J=2.0 \mathrm{~Hz}), 6.38$ $(1 \mathrm{H}, \mathrm{d}, J=2.0 \mathrm{~Hz}), 6.16(1 \mathrm{H}, \mathrm{s}), 5.12(2 \mathrm{H}, \mathrm{s}), 2.48(2 \mathrm{H}, \mathrm{t}, J=7.6 \mathrm{~Hz}), 1.68$ $(2 \mathrm{H}$, app pent, $J=7.5 \mathrm{~Hz}), 1.30-1.36(4 \mathrm{H}, \mathrm{m}), 0.91(3 \mathrm{H}, \mathrm{t}, J=7.0 \mathrm{~Hz}) ;{ }^{13} \mathrm{C}$ NMR $\left(100 \mathrm{MHz}, \mathrm{CDCl}_{3}\right) \delta 166.5,165.8,163.6,158.1,139.5,135.8,130.1$, $128.7,128.4,127.5,103.9,101.8,101.0,70.3,33.3,31.1,26.4,22.3,13.9$.

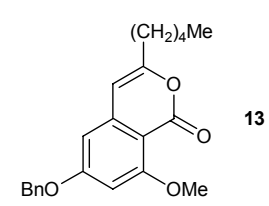

6-Benzyloxy-8-methoxy-3-pentyl-isochromen-1-one (13). IR (film) 2929, $2858,1730,1664,1598,1571,1456,1365,1208,1167,997,692 \mathrm{~cm}^{-1} ;{ }^{1} \mathrm{H}$ NMR $\left(300 \mathrm{MHz}, \mathrm{CDCl}_{3}\right) \delta 7.35-7.45(5 \mathrm{H}, \mathrm{m}), 6.50(1 \mathrm{H}, \mathrm{d}, J=2.4 \mathrm{~Hz}), 6.39$ $(1 \mathrm{H}, \mathrm{d}, J=2.4 \mathrm{~Hz}), 6.07(1 \mathrm{H}, \mathrm{s}), 5.13(2 \mathrm{H}, \mathrm{s}), 3.94(3 \mathrm{H}, \mathrm{s}), 2.44(2 \mathrm{H}, \mathrm{t}, J=7.6$ $\mathrm{Hz}), 1.67(2 \mathrm{H}$, app pent, $J=7.5 \mathrm{~Hz}), 1.25-1.35(4 \mathrm{H}, \mathrm{m}), 0.90(3 \mathrm{H}, \mathrm{t}, J=6.6$ $\mathrm{Hz}) ;{ }^{13} \mathrm{C}$ NMR $\left(75 \mathrm{MHz}, \mathrm{CDCl}_{3}\right) \delta 164.4,163.2,159.6,159.2,142.4,135.7,128.7,128.4,127.5$, $103.1,102.9,100.3,98.7,70.3,56.2,33.2,31.1,26.4,22.3,13.9$.

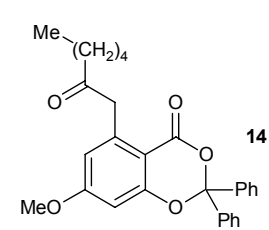

7-Methoxy-5-(2-oxo-heptyl)-2,2-diphenyl-benzo[1,3]dioxin-4-one (14). A mixture of phenol iii (74 mg, $0.172 \mathrm{mmol}, 1 \mathrm{eq}), \mathrm{Me}_{3} \mathrm{OBF}_{4}(127 \mathrm{mg}, 0.86$ $\mathrm{mmol}, 5 \mathrm{eq})$, proton-sponge (221 $\mathrm{mg}, 1.03 \mathrm{mmol}, 6 \mathrm{eq})$ and $4 \AA \mathrm{ms}(328 \mathrm{mg})$ in $\mathrm{CH}_{2} \mathrm{Cl}_{2}(9 \mathrm{~mL})$ was stirred at $\mathrm{rt}$ and under $\mathrm{N}_{2}$ for $16 \mathrm{~h}$, after which the reaction was diluted with EtOAc $(20 \mathrm{~mL})$, filtered through a pad of celite and washed with water $(2 \times 50 \mathrm{~mL})$ and aqueous $\mathrm{CuSO}_{4}(1 \mathrm{M}, 50 \mathrm{~mL})$. The organic phase was dried over $\mathrm{MgSO}_{4}$, concentrated and purified by $\mathrm{FC}$ (silicagel, $25 \%$ EtOAc/Hexanes) to afford $73 \mathrm{mg}$ (95\%) of pure 14: IR (film) 2930, 2360, 2341, 1727, 1614, 1578, 1451, 1273, 1204, $1158,1075,701 \mathrm{~cm}^{-1} ;{ }^{1} \mathrm{H}$ NMR $\left(400 \mathrm{MHz}, \mathrm{CDCl}_{3}\right) \delta$ 7.54-7.56 (4H, dd, $\left.J=1.4 / 8.0 \mathrm{~Hz}\right)$, $7.30-7.36(6 \mathrm{H}, \mathrm{m}), 6.56(1 \mathrm{H}, \mathrm{d}, J=2.4 \mathrm{~Hz}), 6.33(1 \mathrm{H}, \mathrm{d}, J=2.4 \mathrm{~Hz}), 3.96(2 \mathrm{H}, \mathrm{s}), 3.82(3 \mathrm{H}, \mathrm{s})$, $2.47(2 \mathrm{H}, \mathrm{t}, J=7.4 \mathrm{~Hz}), 1.57(2 \mathrm{H}$, app pent, $J=7.2 \mathrm{~Hz}), 1.21-1.33(4 \mathrm{H}, \mathrm{m}), 0.88(3 \mathrm{H}, \mathrm{t}, J=7.2$ $\mathrm{Hz}) ;{ }^{13} \mathrm{C}$ NMR $\left(100 \mathrm{MHz}, \mathrm{CDCl}_{3}\right) \delta 206.8,165.0,160.5,159.3,141.2,139.7,129.1,128.4$, 126.4 114.4, 106.4, 106.0, 100.8, 55.6, 48.4, 42.4, 31.3, 23.2, 22.4, 13.9; LRMS (ESI) Calcd for $\mathrm{C}_{28} \mathrm{H}_{28} \mathrm{NaO}_{5}$ [MNa] $]^{+}$467.18; Found: 467.15. HRMS (ESI, added $\mathrm{NaO}_{2} \mathrm{CCF}_{3}$ ) Calcd for $\mathrm{C}_{28} \mathrm{H}_{28} \mathrm{O}_{5} \mathrm{Na}[\mathrm{MNa}]^{+}: 467.1834$. Found: 467.1823.

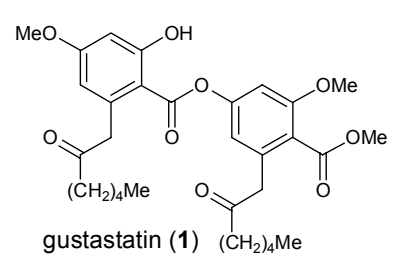

Gustastatin (1). A solution of phenol $11(50 \mathrm{mg}, 0.170 \mathrm{mmol}, 1 \mathrm{eq})$ and benzodioxinone $14(151 \mathrm{mg}, 0.340 \mathrm{mmol}, 2 \mathrm{eq})$ in benzene $(5 \mathrm{~mL})$ was concentrated on the rotavapor ( 3 times). The residue was dissolved in freshly distilled $\mathrm{CH}_{2} \mathrm{Cl}_{2}(1.7 \mathrm{~mL})$ and transferred to an oven-dried borosilicate test tube sealed with a rubber septum under nitrogen inlet. The nitrogen inlet was removed and the reaction vessel was sealed with parafilm. This solution was then photolyzed in a Rayonett reactor for $2 \mathrm{~h}$ at $300 \mathrm{~nm}$. The solution was concentrated and purified by FC (silicagel, $15 \rightarrow 30 \%$ EtOAc/Hexanes) to afford $42.1 \mathrm{mg}$ (28\%) of recovered benzodioxinone 14 and $66.7 \mathrm{mg}$ (71\%) of pure gustastatin (1) as an amorphous solid: $\mathrm{mp} 134-138^{\circ} \mathrm{C}$; UV $\left(\mathrm{CHCl}_{3}\right) 246.0,272.5$, 303.5; IR (film) 2950, 2932, 1715, $1657,1597,1461,1428,1332,1256,1160,114.6,1074,1042,951,822,740 \mathrm{~cm}^{-1} ;{ }^{1} \mathrm{H}$ NMR (400 $\left.\mathrm{MHz}, \mathrm{CDCl}_{3}\right) \delta 11.27(1 \mathrm{H}, \mathrm{s}), 6.59(1 \mathrm{H}, \mathrm{d}, J=1.6 \mathrm{~Hz}), 6.56(1 \mathrm{H}, \mathrm{d}, J=1.2 \mathrm{~Hz}), 6.46(1 \mathrm{H}, \mathrm{d}, J=$ 
$2.4 \mathrm{~Hz}), 6.29(1 \mathrm{H}, \mathrm{d}, J=2.4 \mathrm{~Hz}), 4.06(2 \mathrm{H}, \mathrm{s}), 3.87(3 \mathrm{H}, \mathrm{s}), 3.84(3 \mathrm{H}, \mathrm{s}), 3.82(3 \mathrm{H}, \mathrm{s}), 3.70(2 \mathrm{H}$, s), $2.44(2 \mathrm{H}, \mathrm{t}, J=7.6 \mathrm{~Hz}), 2.41(2 \mathrm{H}, \mathrm{t}, J=7.6 \mathrm{~Hz}), 1.51-1.59(4 \mathrm{H}, \mathrm{m}), 1.26(4 \mathrm{H}, \mathrm{m}), 1.22(4 \mathrm{H}$, m), $0.88(3 \mathrm{H}, \mathrm{t}, J=7.0 \mathrm{~Hz}), 0.83(3 \mathrm{H}, \mathrm{t}, J=7.0 \mathrm{~Hz}) ;{ }^{13} \mathrm{C} \mathrm{NMR}\left(75 \mathrm{MHz}, \mathrm{CDCl}_{3}\right) \delta 207.4,206.5$, 169.1, 167.4, 166.5, 164.9, 158.3, 151.2, 138.9, 135.4, 121.7, 116.1, 113.3, 104.4, 104.2, 100.0, 56.2, 55.5, 52.3, 51.2, 47.5, 42.5, 42.1, 31.3, 31.2, 23.4, 23.3, 22.4, 13.9, 13.8; LRMS (ESI) Calcd for $\mathrm{C}_{31} \mathrm{H}_{40} \mathrm{O}_{9} \mathrm{Na}[\mathrm{M}+\mathrm{Na}]^{+}$: 579.26; Found: 579.20; Calcd for $\mathrm{C}_{31} \mathrm{H}_{39} \mathrm{O}_{9}[\mathrm{M}-\mathrm{H}]^{-}: 555.26$; Found: 555.35. HRMS (ESI, added $\mathrm{NaO}_{2} \mathrm{CCF}_{3}$ ) Calcd for $\mathrm{C}_{31} \mathrm{H}_{40} \mathrm{O}_{9} \mathrm{Na}[\mathrm{MNa}]^{+}: 579.2570$. Found: 579.2587. Elemental Anal. Calcd for $\mathrm{C}_{31} \mathrm{H}_{40} \mathrm{O}_{9}$ : C, 66.89; H, 7.24; O, 25.87. Found: C, $67.07 ; \mathrm{H}, 7.07 ; \mathrm{O}, 25.19$.

\section{Data comparation of natural and synthetic gustastatin.}

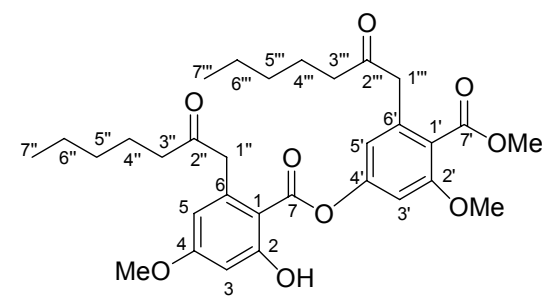

Table S1: ${ }^{1} \mathrm{H}$ NMR Data for natural $\left(500 \mathrm{MHz}, \mathrm{CDCl}_{3}\right)$ and synthetic $\left(400 \mathrm{MHz}, \mathrm{CDCl}_{3}\right)$ gustastatin (1).

\begin{tabular}{|c|c|c|}
\hline \multirow[b]{2}{*}{ Position } & \multicolumn{2}{|c|}{$\delta$ in ppm (multiplicity, $J$ in $\mathrm{Hz}$ ) } \\
\hline & Natural 1 & Synthetic 1 \\
\hline $\mathrm{OH} 2$ & $11.28(\mathrm{~s})$ & $11.27(\mathrm{~s})$ \\
\hline H3' & $6.59(\mathrm{~d}, 1.5)$ & $6.59(\mathrm{~d}, 1.6)$ \\
\hline $\mathrm{H} 5$, & $6.57(\mathrm{~d}, 1.5)$ & $6.56(\mathrm{~d}, 1.2)$ \\
\hline $\mathrm{H} 3$ & $6.46(\mathrm{~d}, 2.5)$ & $6.46(\mathrm{~d}, 2.4)$ \\
\hline H5 & $6.29(\mathrm{~d}, 2.5)$ & $6.29(\mathrm{~d}, 2.4)$ \\
\hline $\mathrm{H} 1$ ', & $4.07(\mathrm{~s})$ & $4.06(\mathrm{~s})$ \\
\hline $\mathrm{CO}_{2} \mathrm{CH}_{3}$ & $3.88(\mathrm{~s})$ & $3.87(\mathrm{~s})$ \\
\hline $4-\mathrm{OCH}_{3}$ & $3.85(\mathrm{~s})$ & $3.84(\mathrm{~s})$ \\
\hline 2'- $-\mathrm{OCH}_{3}$ & $3.83(\mathrm{~s})$ & $3.82(\mathrm{~s})$ \\
\hline $\mathrm{H} 1{ }^{\prime,}$ & $3.70(\mathrm{~s})$ & $3.70(\mathrm{~s})$ \\
\hline H3', & $2.44(\mathrm{t}, 7.5)$ & $2.44(t, 7.6)$ \\
\hline $\mathrm{H} 3^{\prime}$, & $2.41(\mathrm{t}, 7.5)$ & $2.41(\mathrm{t}, 7.6)$ \\
\hline $\mathrm{H} 4$ ", & 1.56 (pent, 7.5 ) & \\
\hline H4,", & 1.53 (pent, 7.5 ) & $1.51-1.59(\mathrm{~m})$ \\
\hline H6”, H6',' & $1.27(\mathrm{~m})$ & $1.26(\mathrm{~m})$ \\
\hline H5', H5,', & $1.21(\mathrm{~m})$ & $1.22(\mathrm{~m})$ \\
\hline $\mathrm{H} 7$ "' and $\mathrm{H} 7$ ', & not reported & $0.88(\mathrm{t}, 7.0) / 0.83(\mathrm{t}, 7.0)$ \\
\hline
\end{tabular}


Table S2: ${ }^{13} \mathrm{C}$ NMR Data for natural $\left(125 \mathrm{MHz}, \mathrm{CDCl}_{3}\right)$ and synthetic $\left(75 \mathrm{MHz}, \mathrm{CDCl}_{3}\right)$ gustastatin (1).

\begin{tabular}{|c|c|c|}
\hline \multirow{2}{*}{ Position } & \multicolumn{2}{|c|}{$\delta$ in ppm } \\
\cline { 2 - 3 } C2'” & Natural 1 & Synthetic 1 \\
\hline C2 $^{\prime}{ }^{\prime}$ & 207.4 & 207.4 \\
\hline C7 & 206.4 & 206.5 \\
\hline C7' & 169.1 & 169.1 \\
\hline C2 & 167.5 & 167.4 \\
\hline C4 & 166.5 & 166.5 \\
\hline C2' & 164.9 & 164.9 \\
\hline C4' & 158.4 & 158.3 \\
\hline C6 & 131.3 & 151.2 \\
\hline C6 & 135.4 & 138.9 \\
\hline C1 & 121.8 & 135.4 \\
\hline C5' & 116.2 & 121.7 \\
\hline C5 & 113.4 & 116.1 \\
\hline C3 & 104.5 & 113.3 \\
\hline C1 & 104.3 & 104.4 \\
\hline
\end{tabular}

\begin{tabular}{|c|c|c|}
\hline \multirow[b]{2}{*}{ Position } & \multicolumn{2}{|c|}{$\delta$ in ppm } \\
\hline & Natural 1 & Synthetic 1 \\
\hline C3 & 100.1 & 100.0 \\
\hline $2^{\prime}-\mathrm{OCH}_{3}$ & 56.3 & 56.2 \\
\hline $4-\mathrm{OCH}_{3}$ & 55.5 & 55.5 \\
\hline $\mathrm{CO}_{2} \mathrm{CH}_{3}$ & 52.3 & 52.3 \\
\hline $\mathrm{C} 1 \%$ & 51.2 & 51.2 \\
\hline $\mathrm{C} 1, "$, & 47.5 & 47.5 \\
\hline C3" & 42.5 & 42.5 \\
\hline C3"” & 42.2 & 42.1 \\
\hline C5" & 31.3 & 31.3 \\
\hline $\mathrm{C} 5, "$ & 31.3 & 31.2 \\
\hline C4", & 23.4 & 23.4 \\
\hline C4", & 23.3 & 23.3 \\
\hline C6" & 22.4 & 22.4 \\
\hline C6"” & 22.4 & 22.4 \\
\hline C7" & 13.9 & 13.9 \\
\hline C7," & 13.8 & 13.8 \\
\hline
\end{tabular}




\section{Proton and Carbon NMR Spectra.}
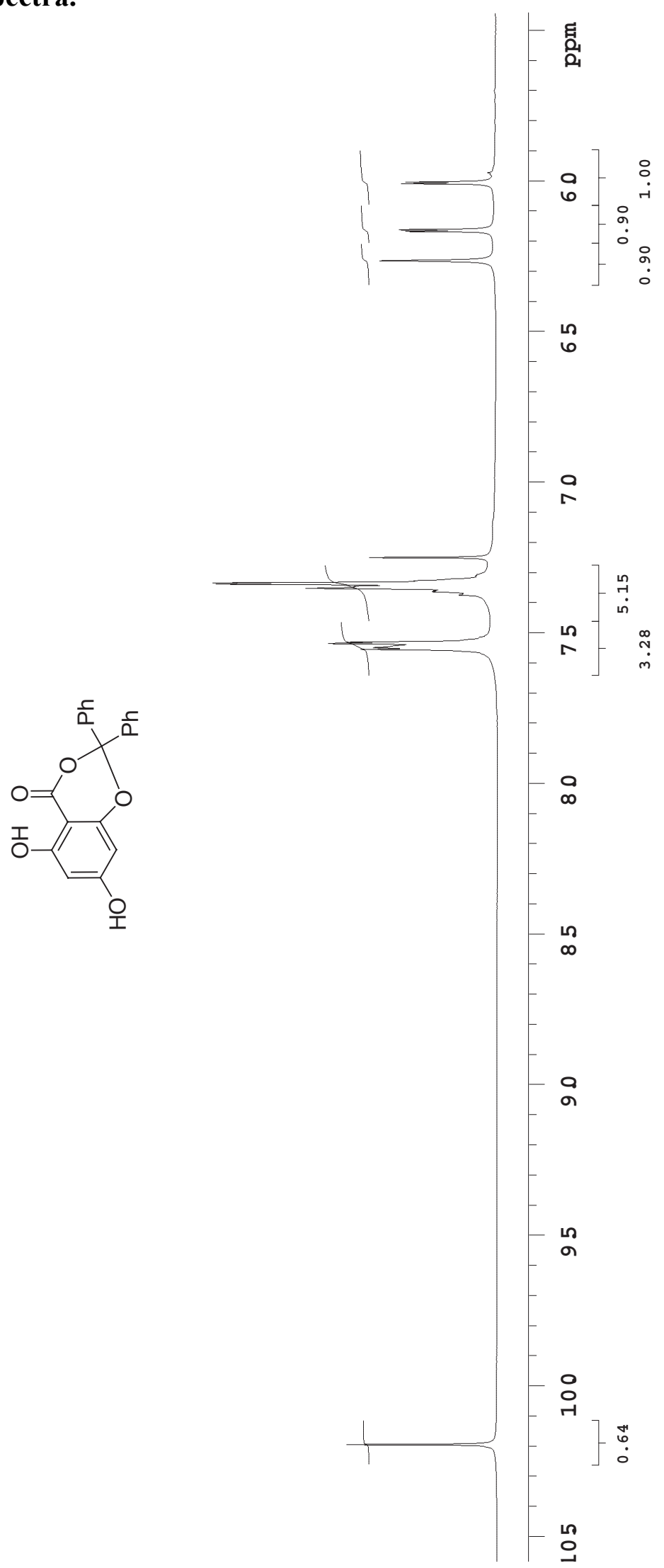


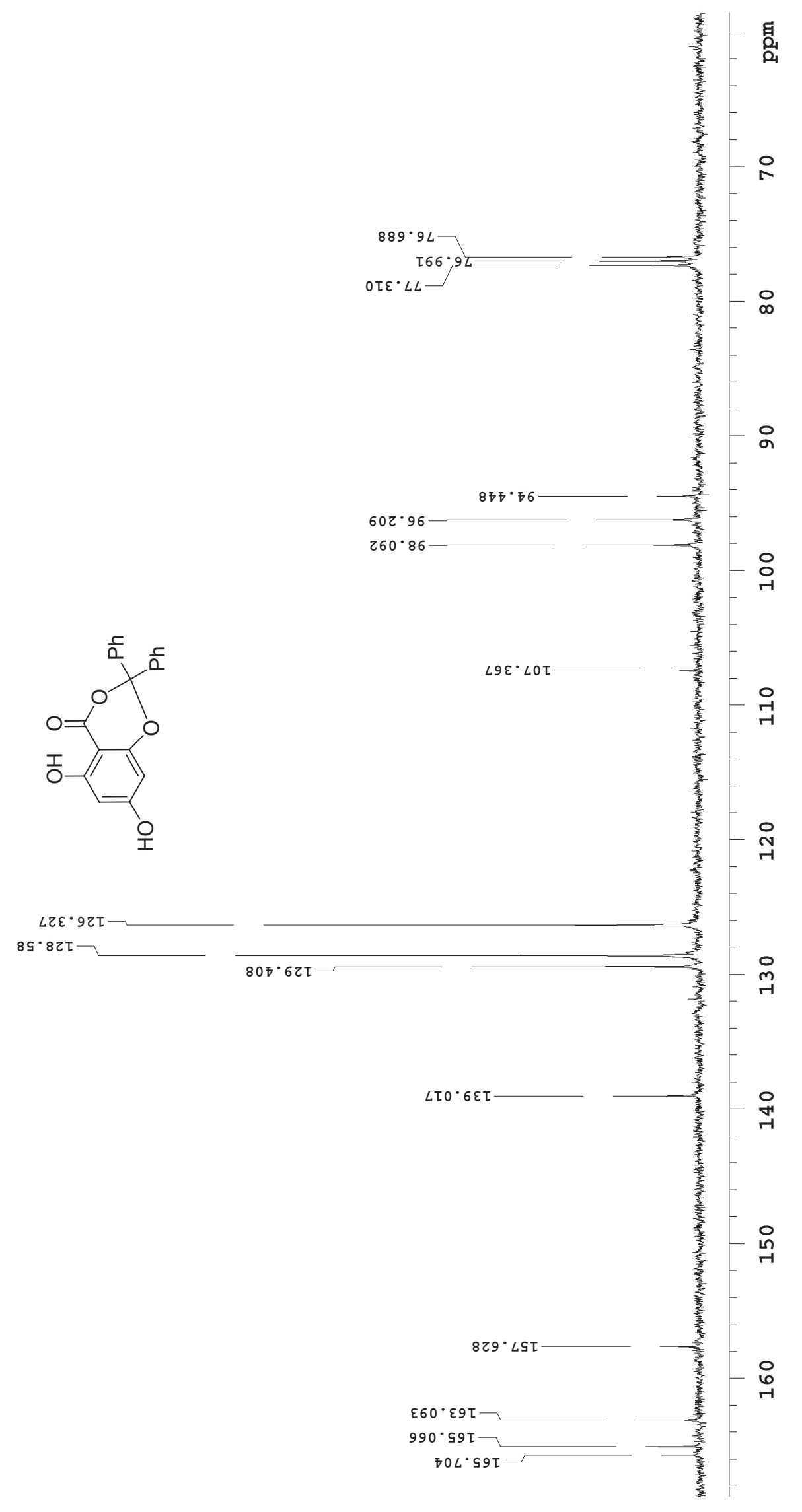

-S10- 


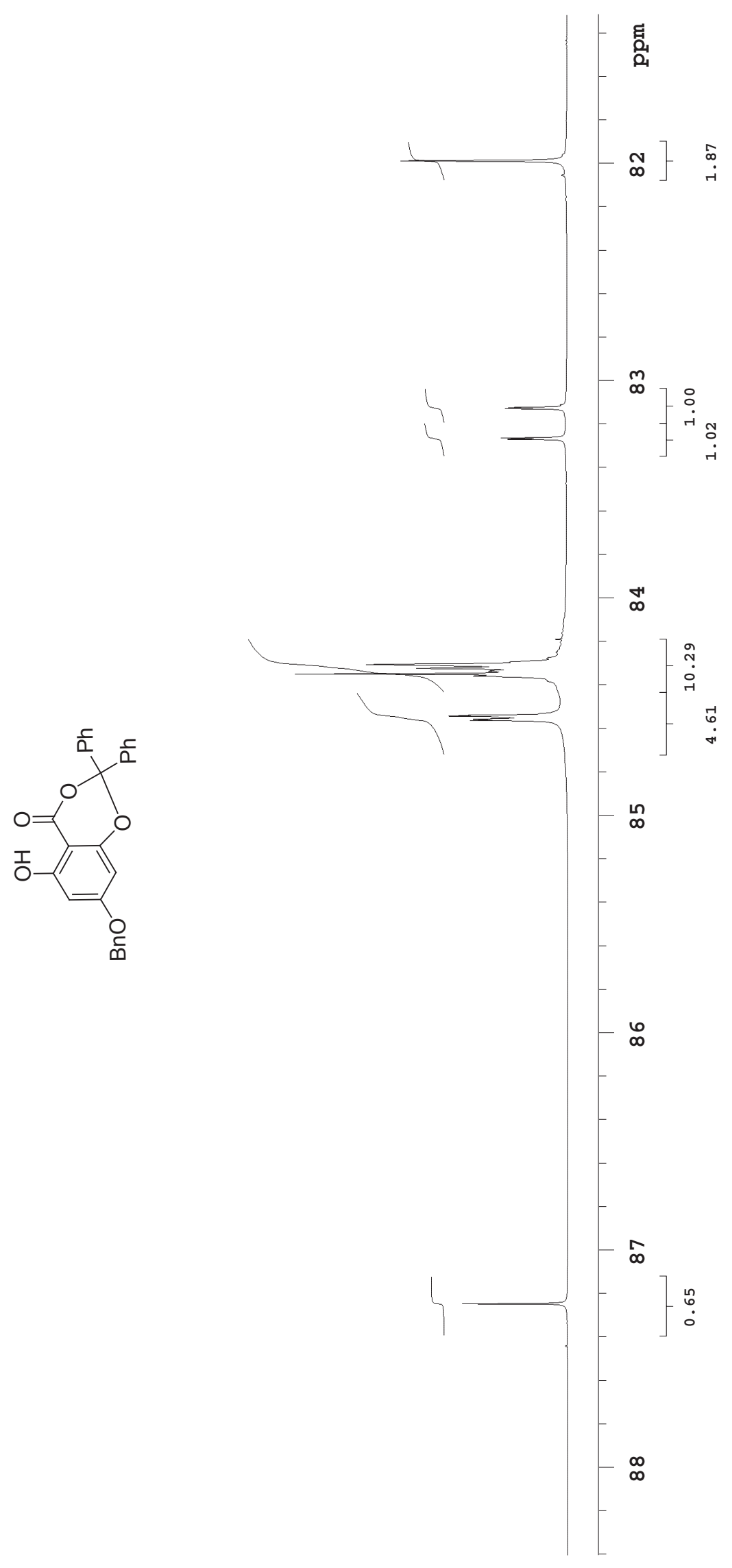

-S11- 


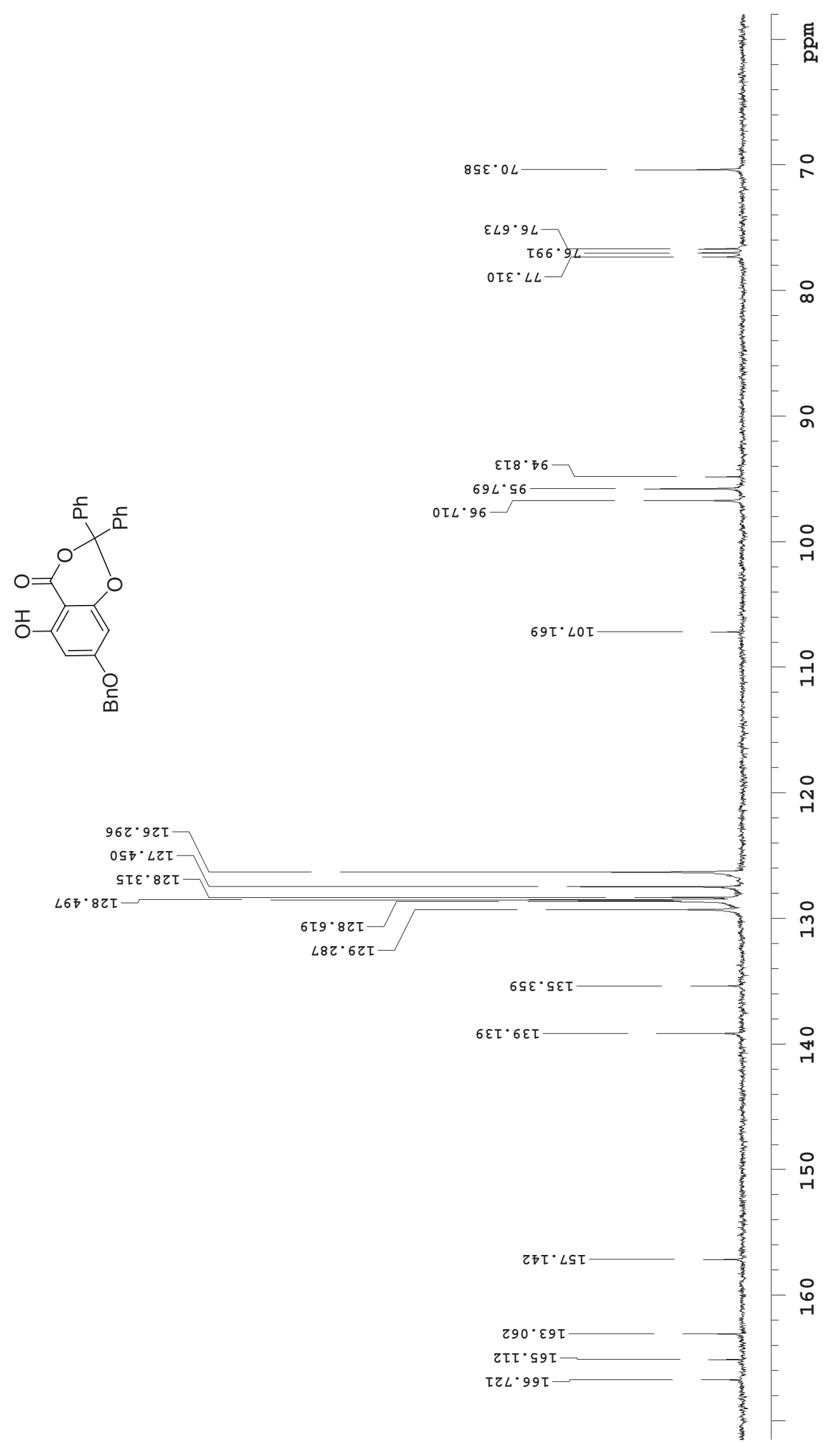

-S12- 


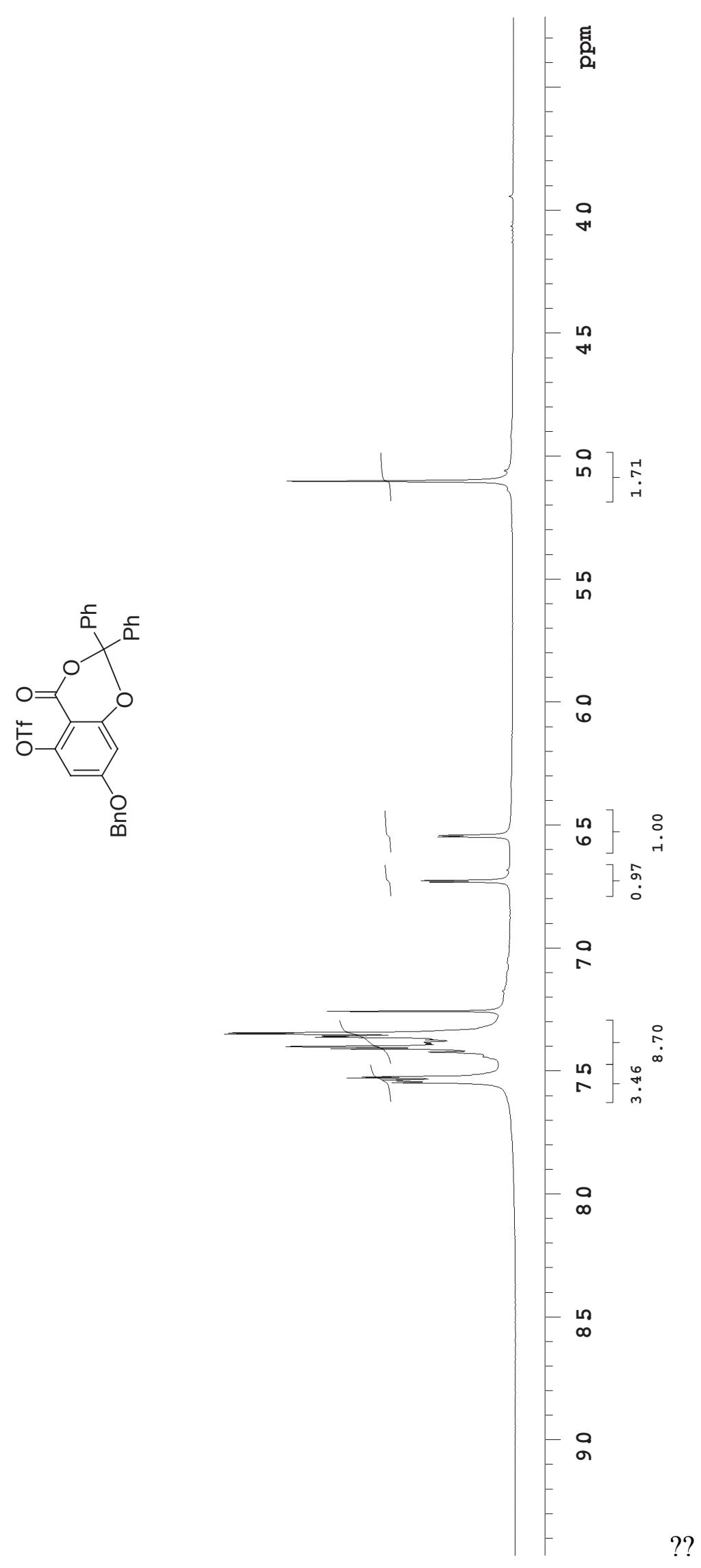

-S13- 


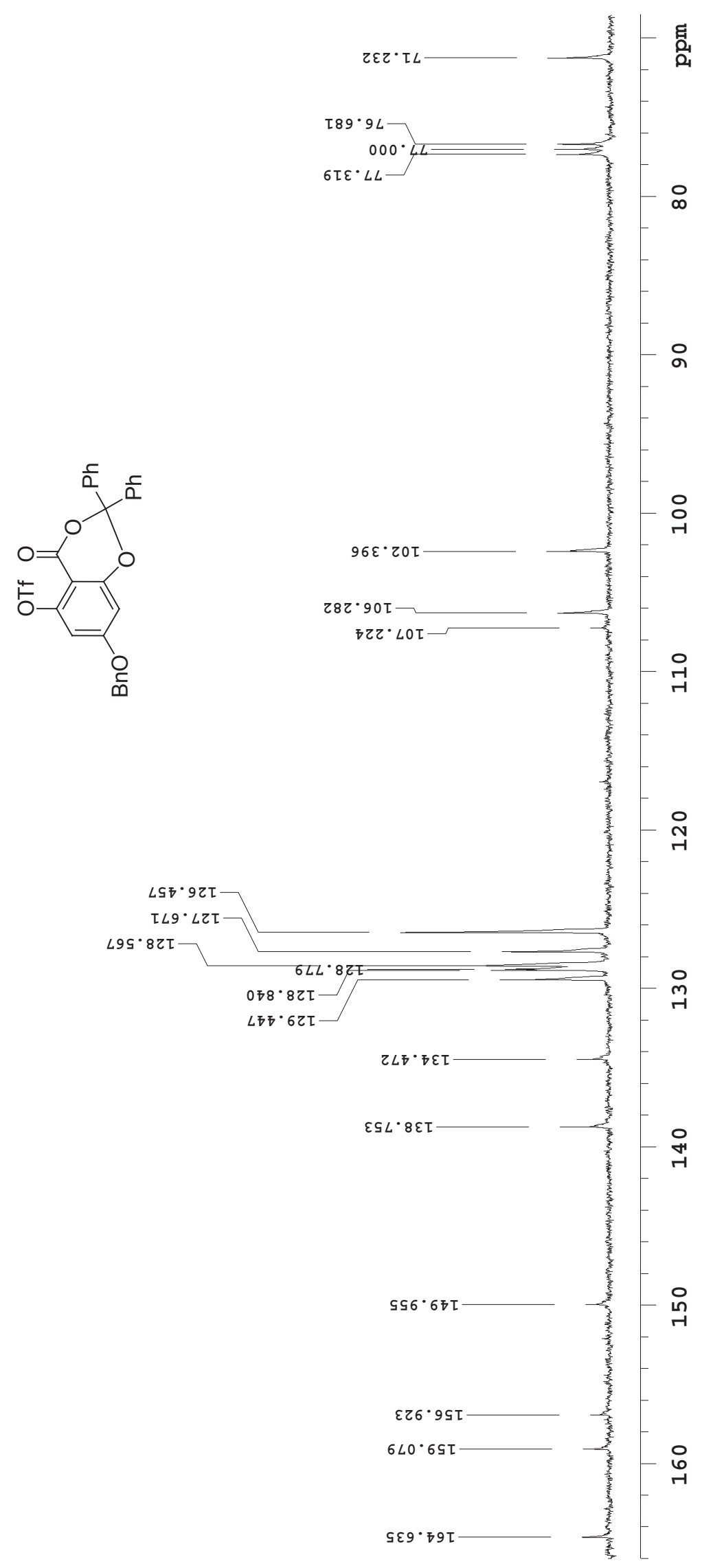




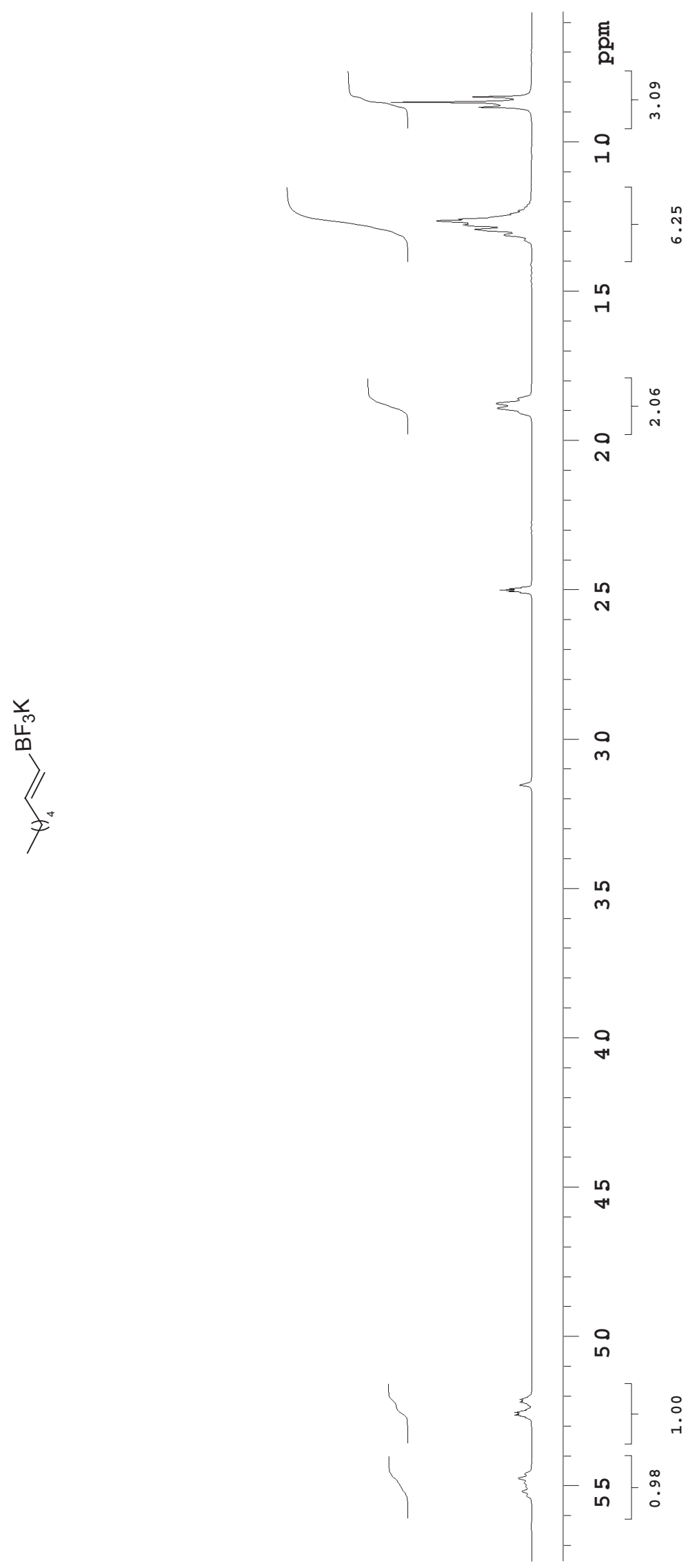

-S15- 


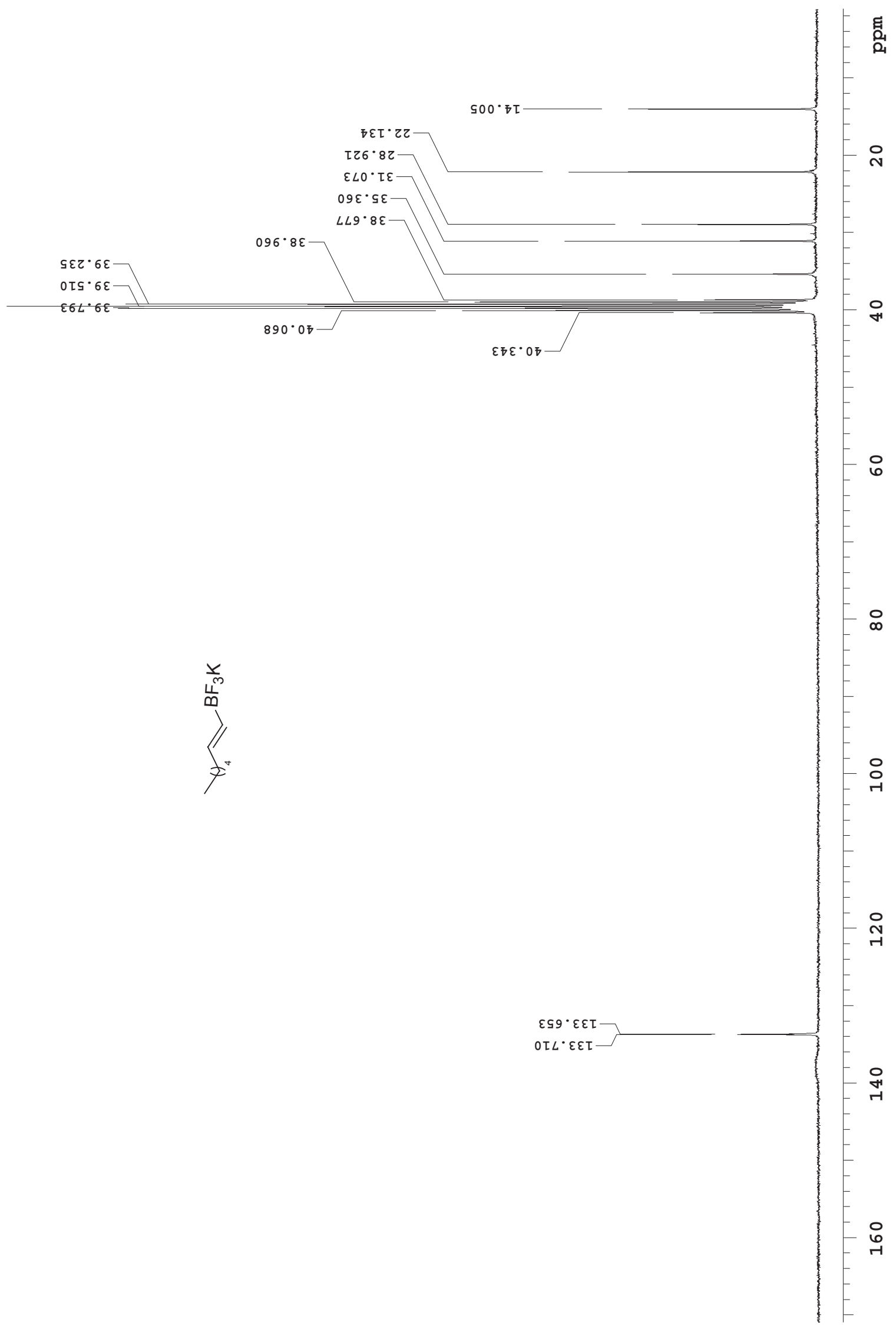




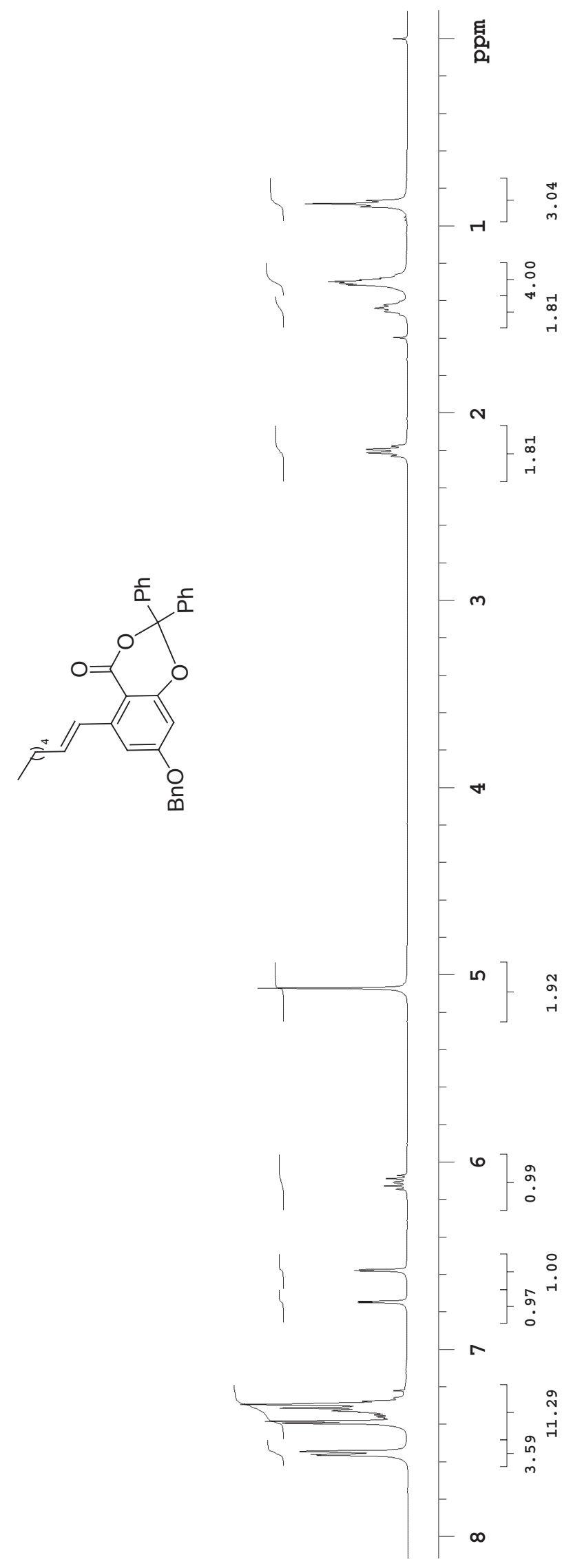

-S17- 


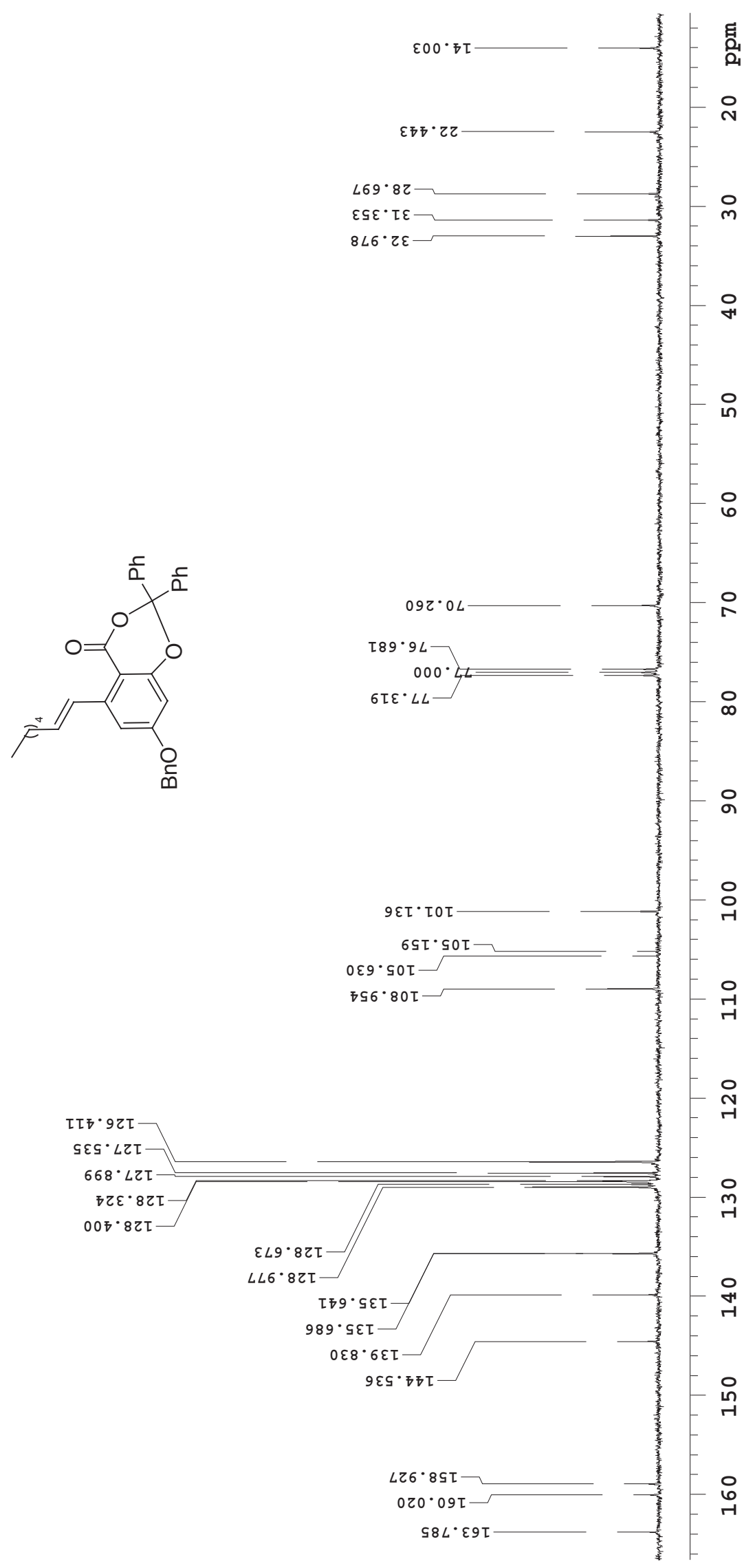

-S18- 


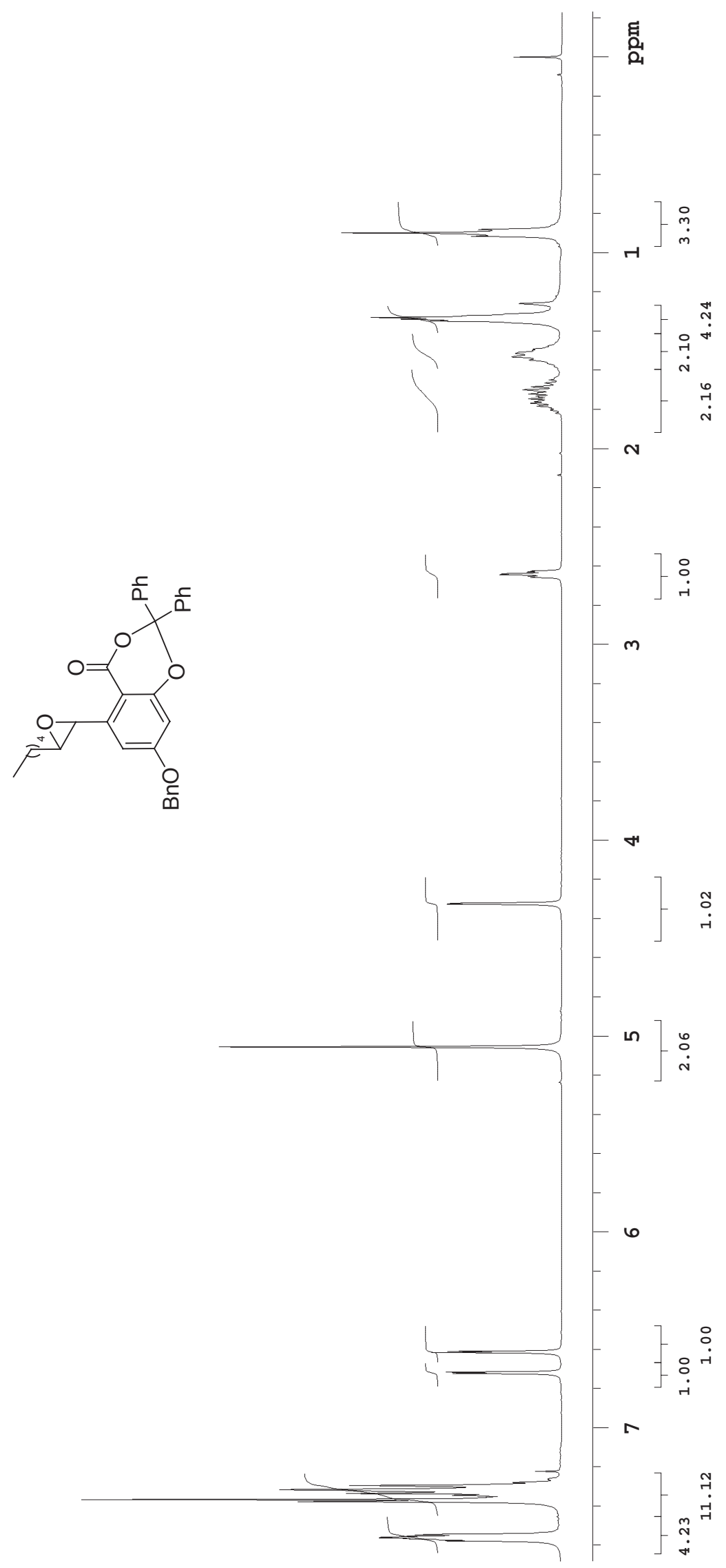

-S19- 


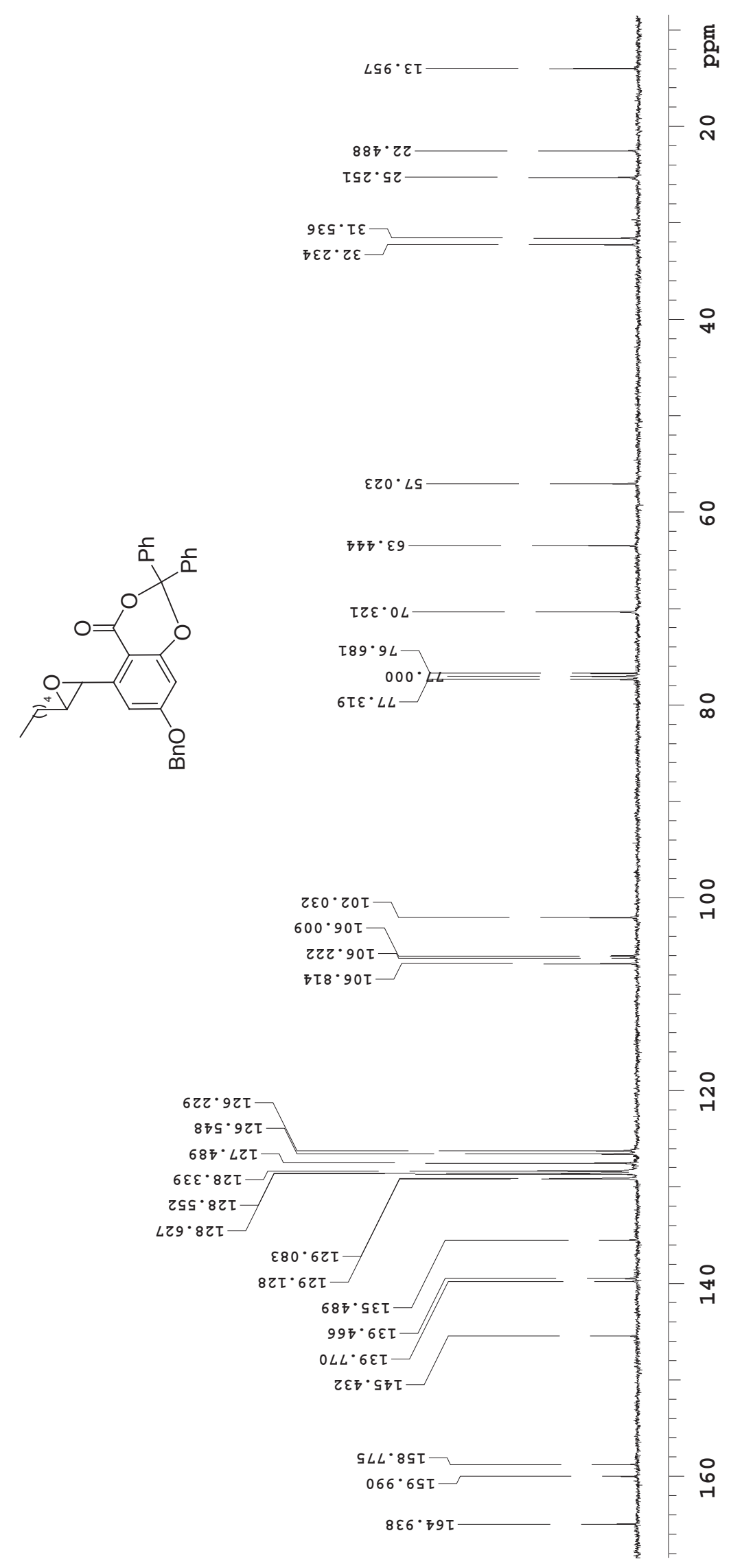




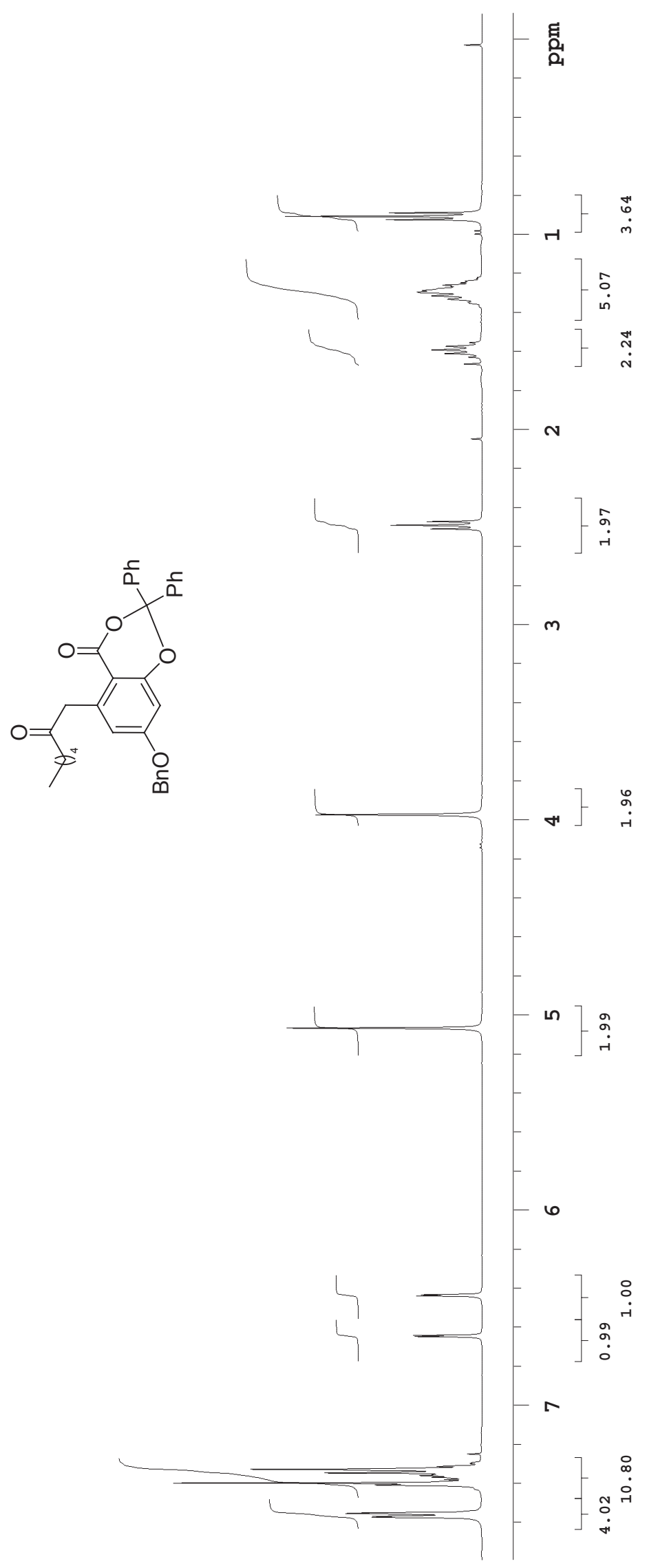




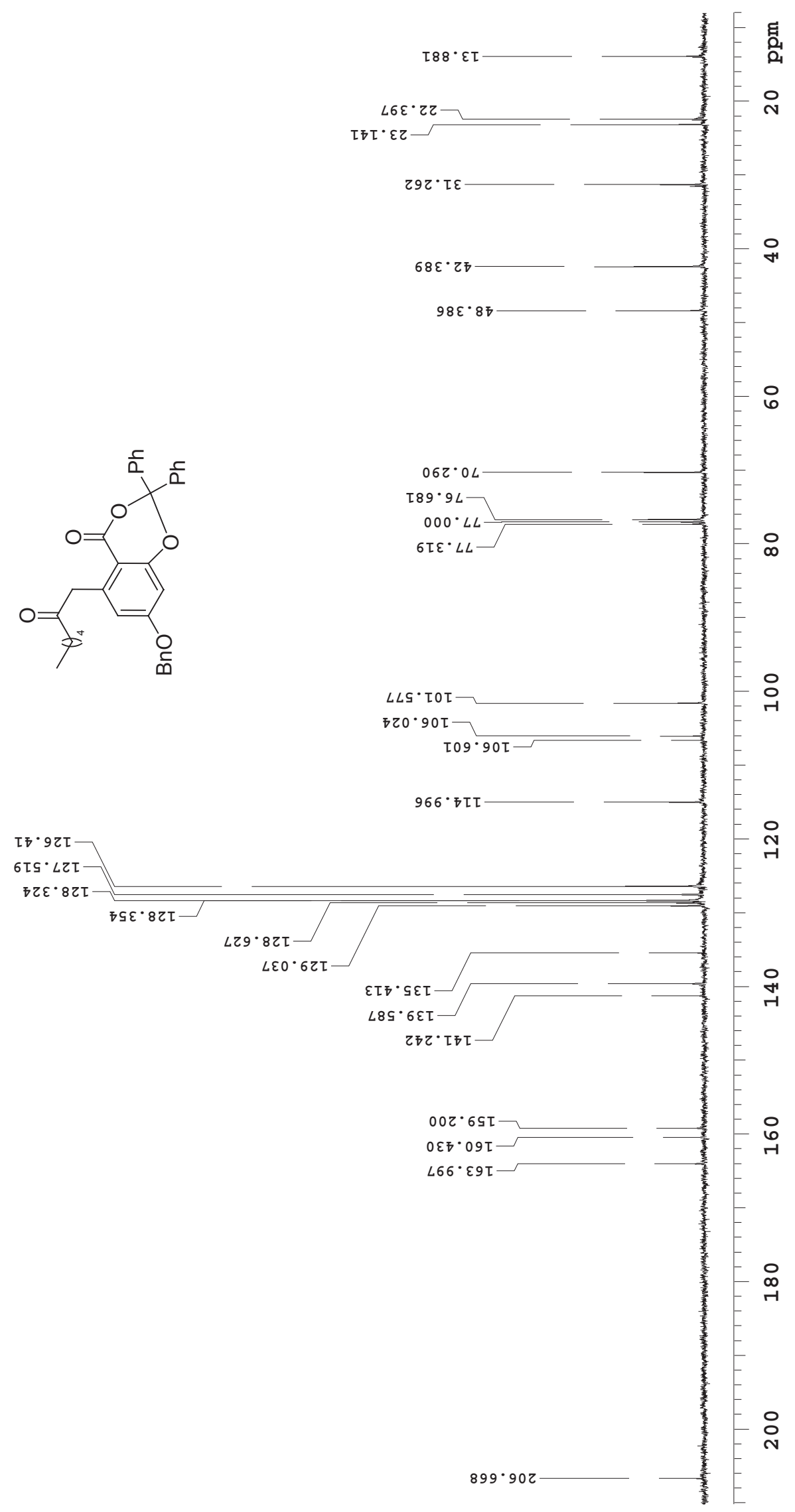




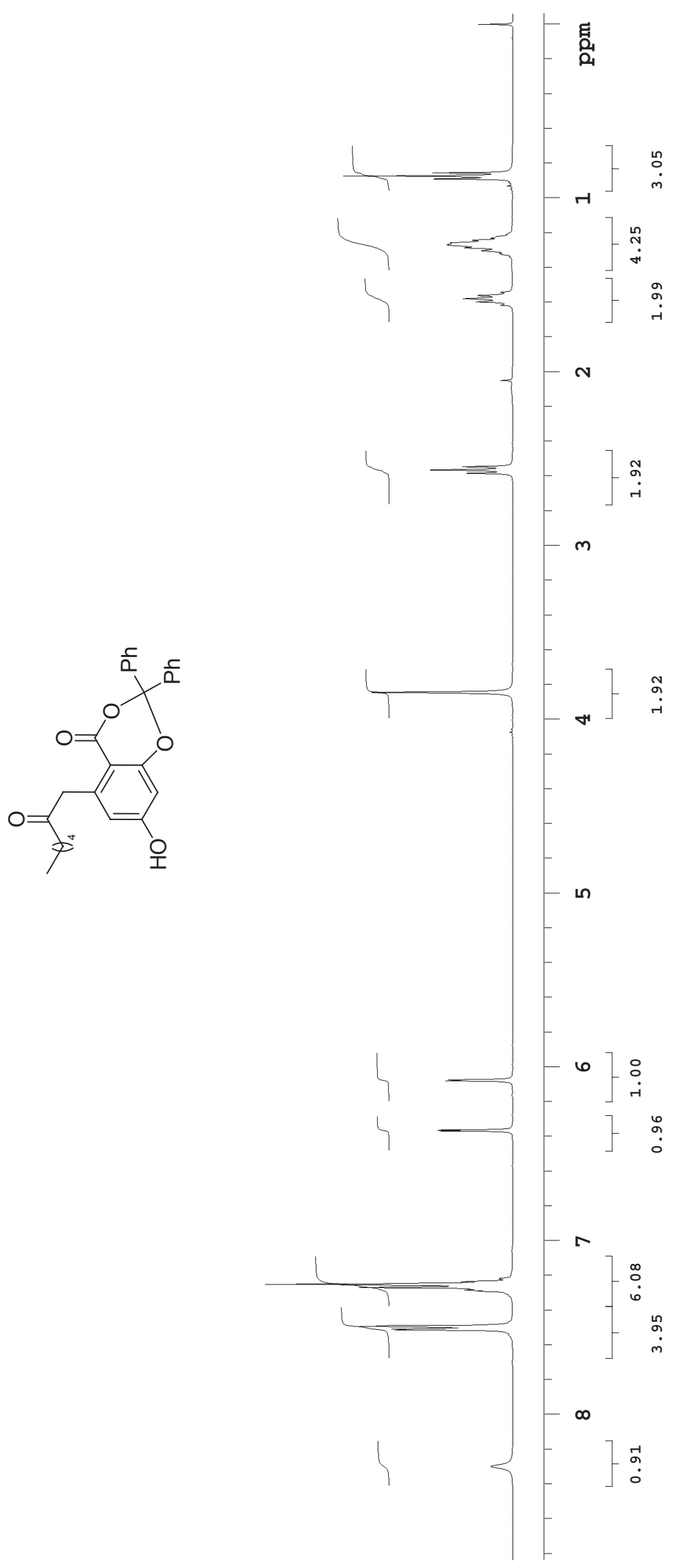




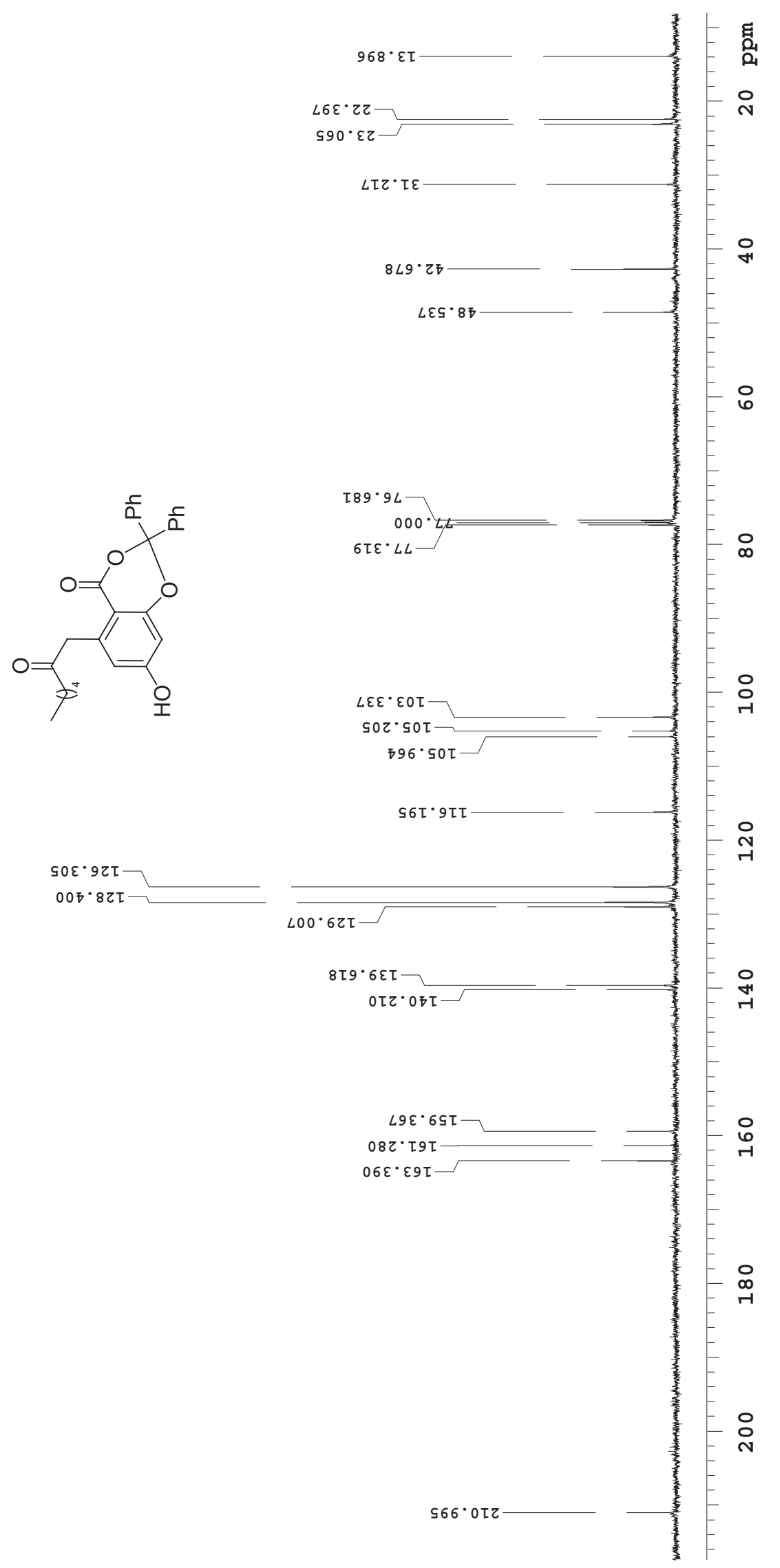




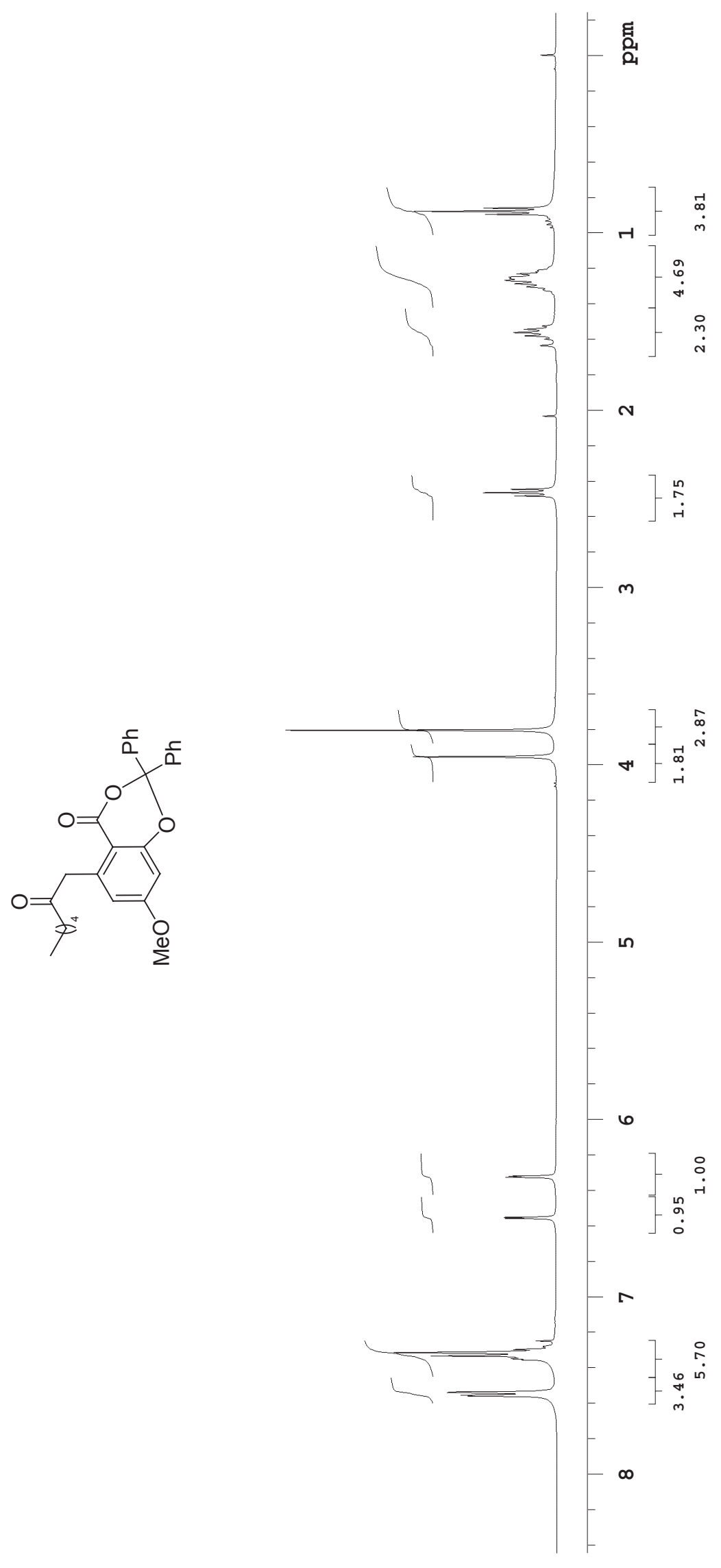



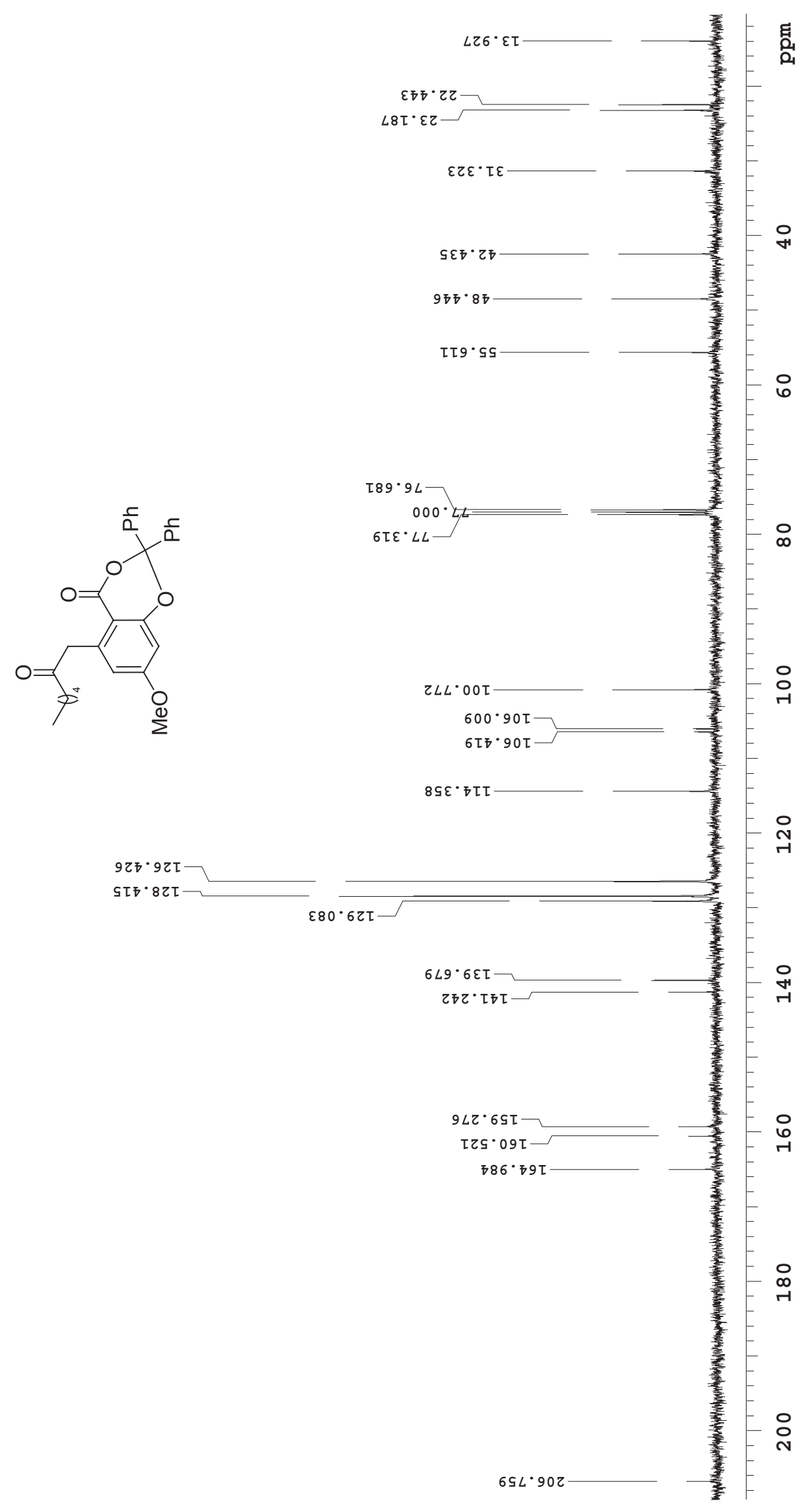


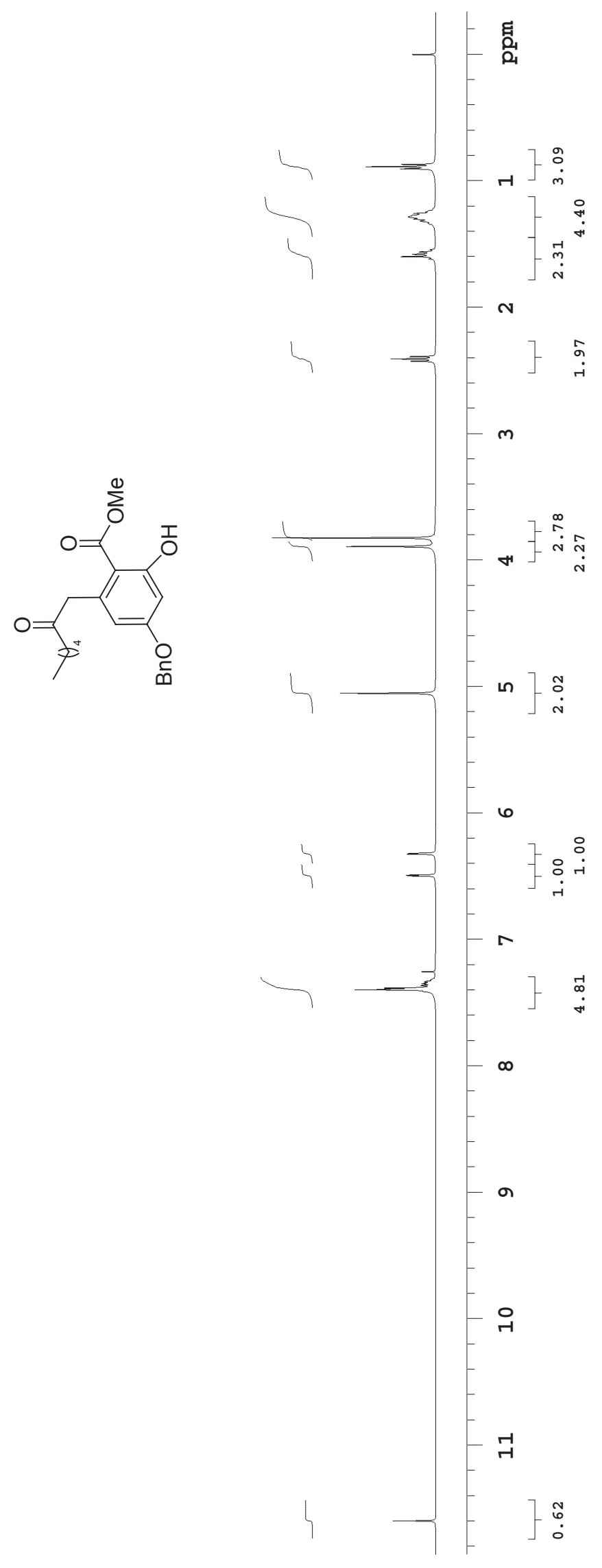




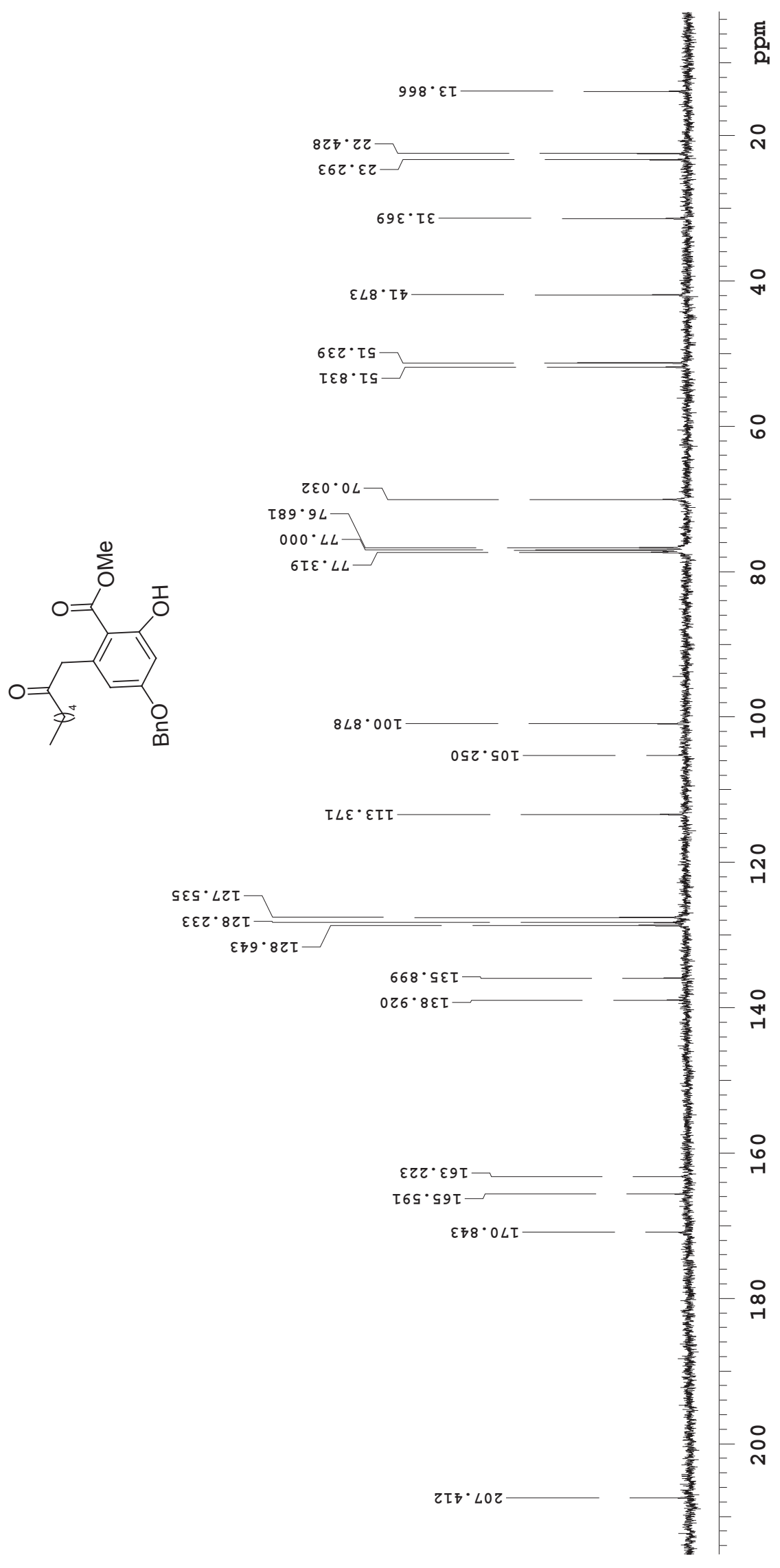




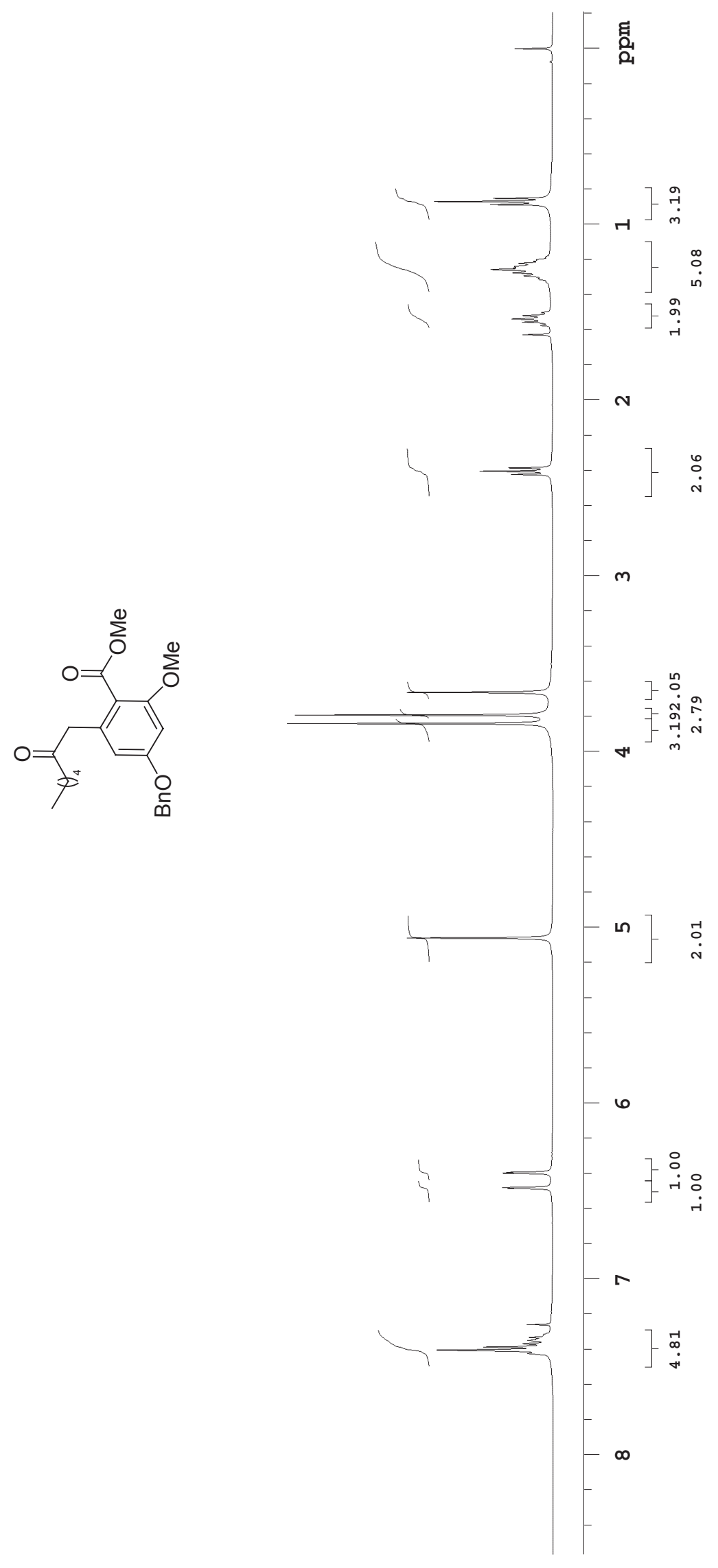




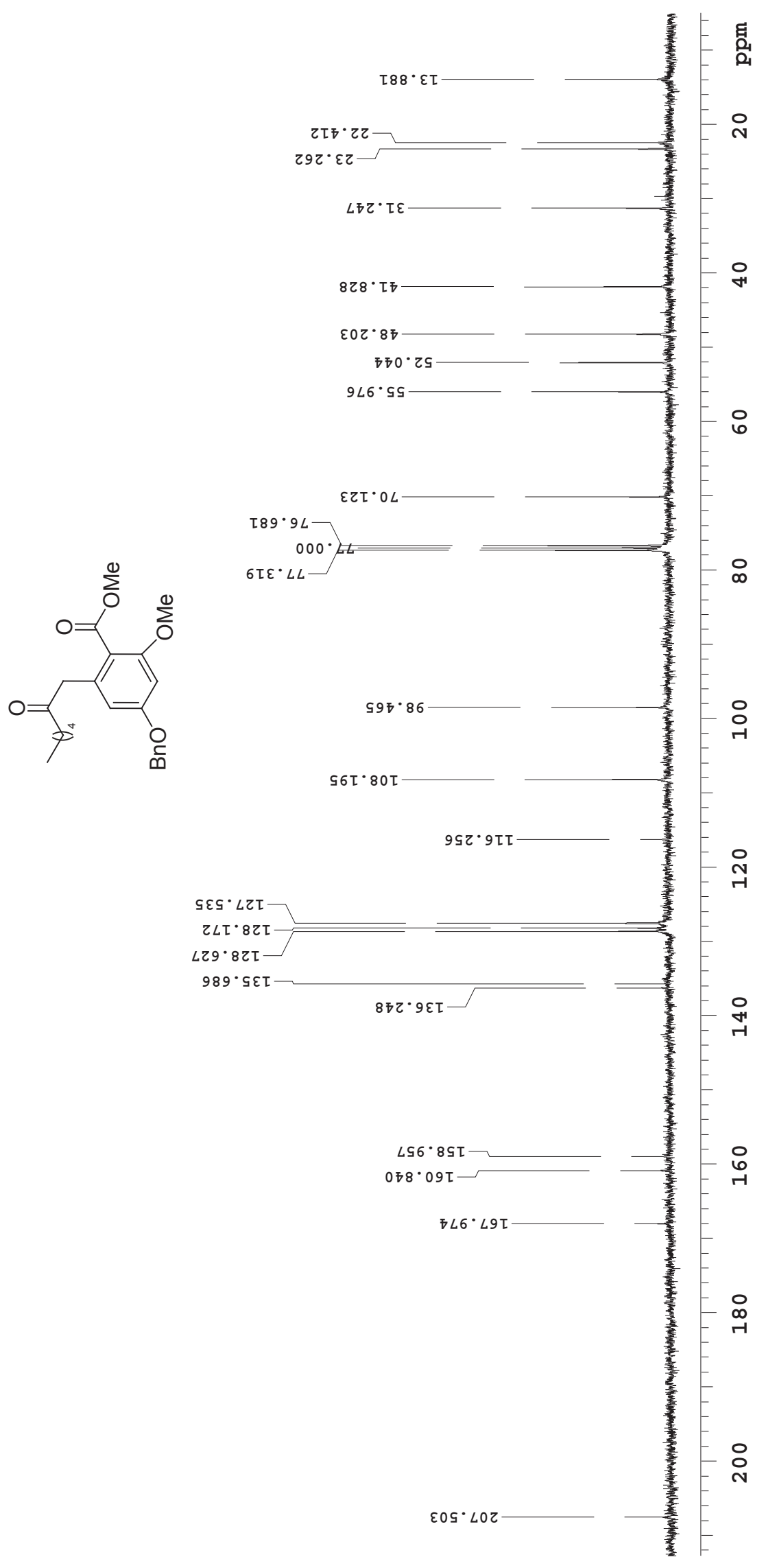




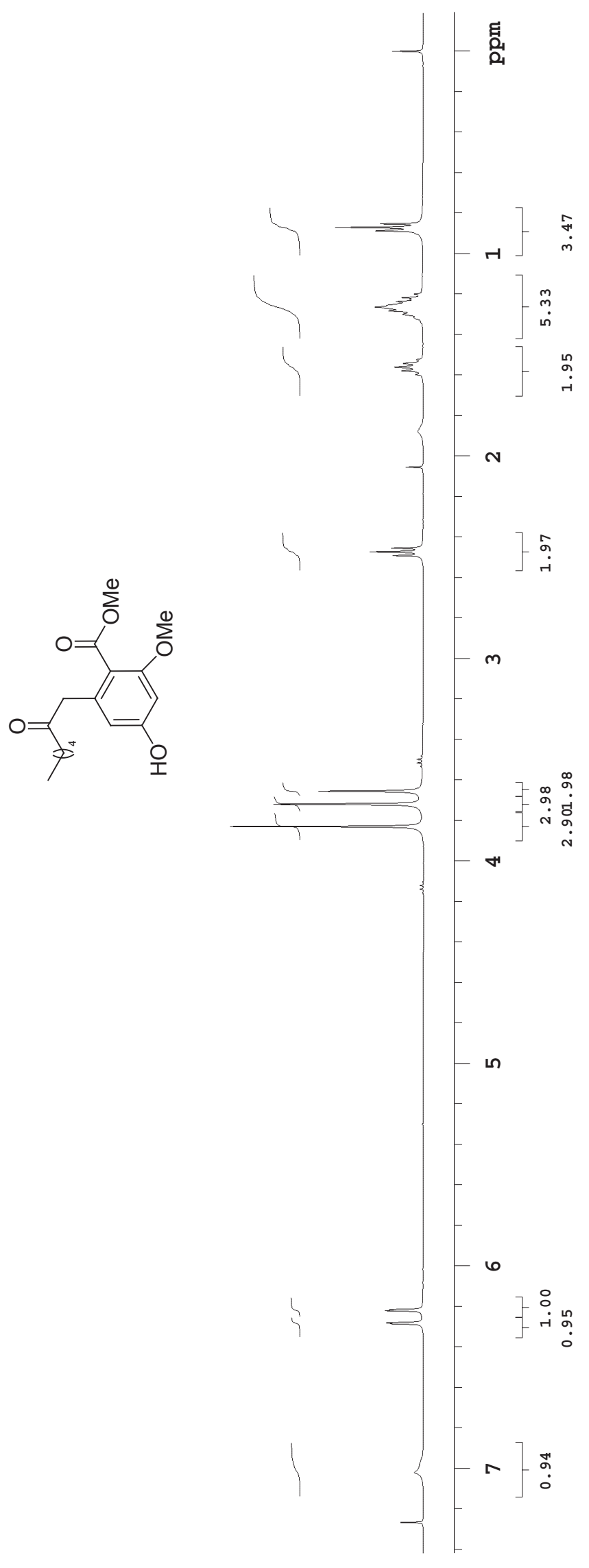




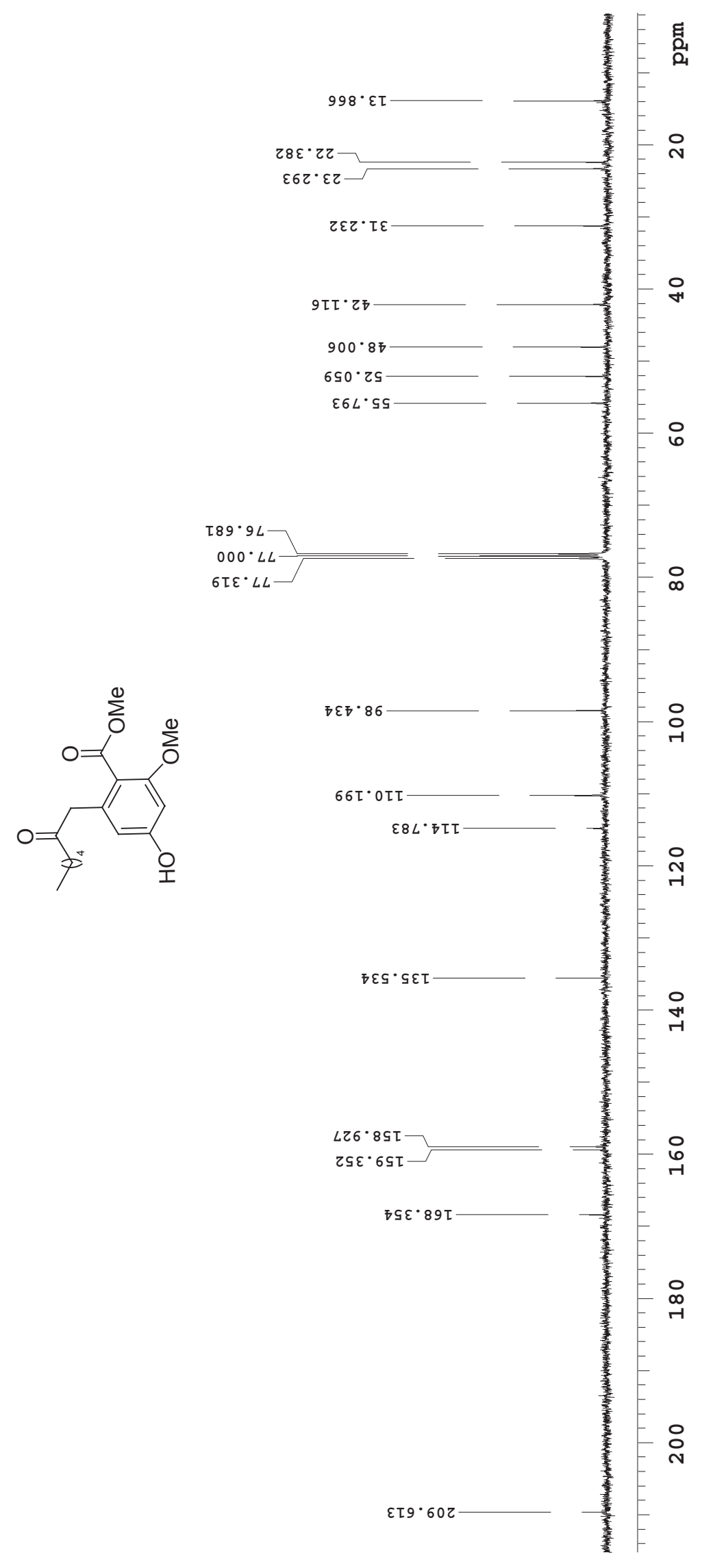




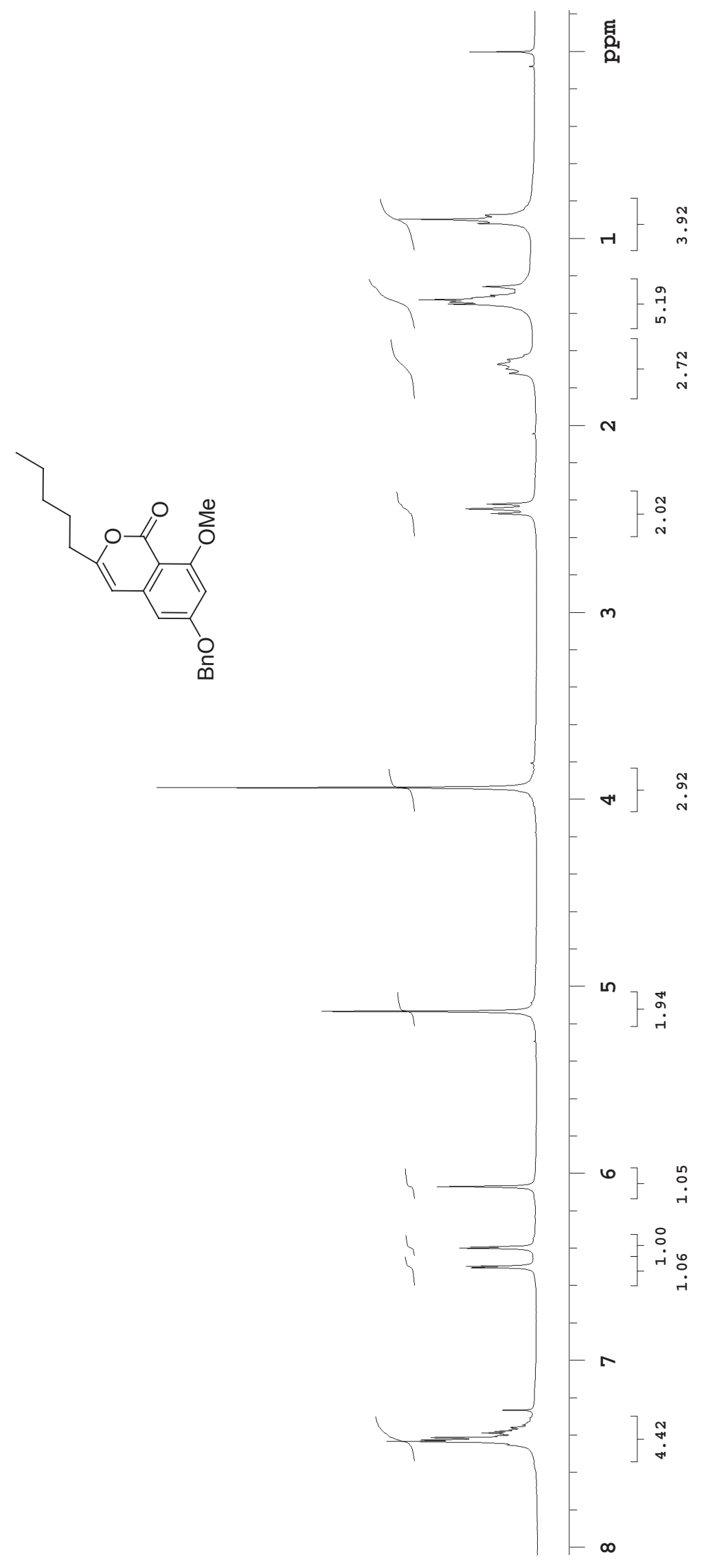

-S33- 


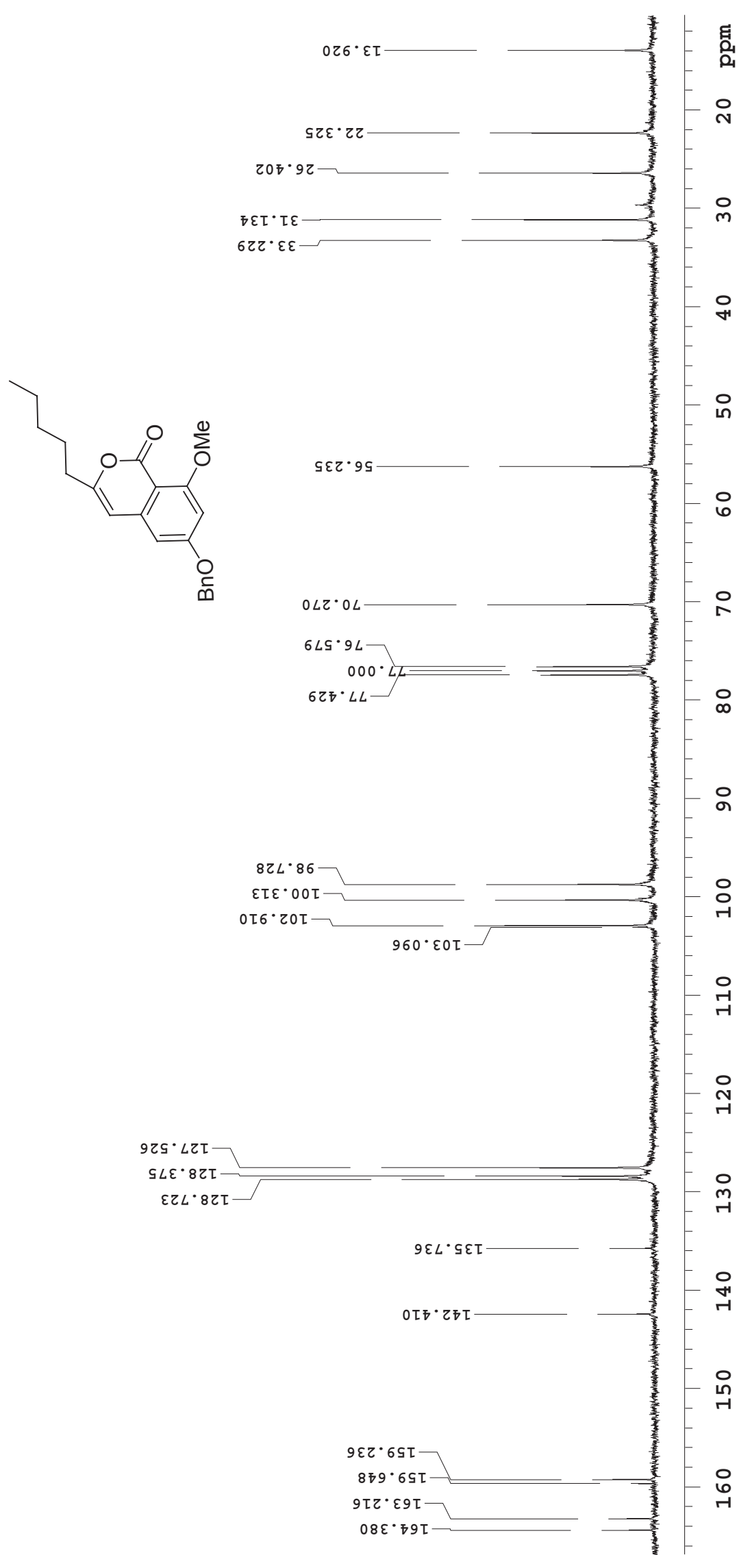




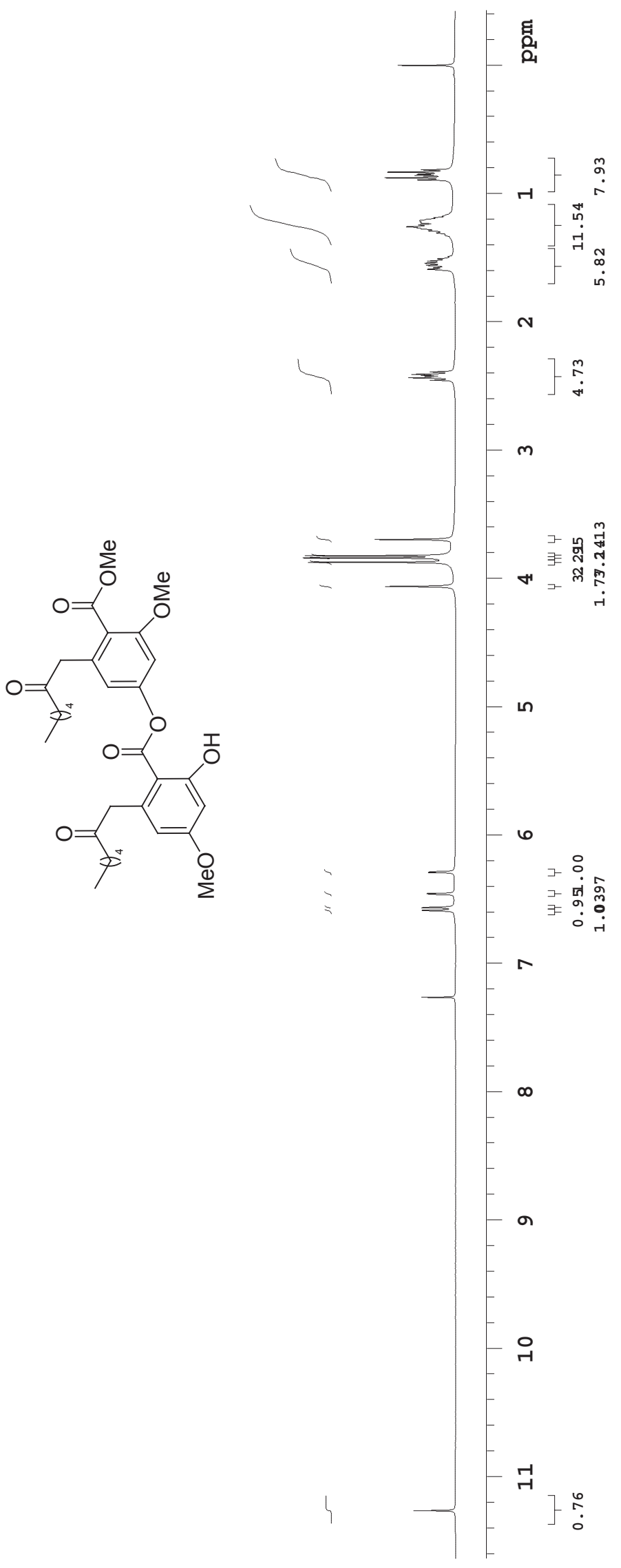




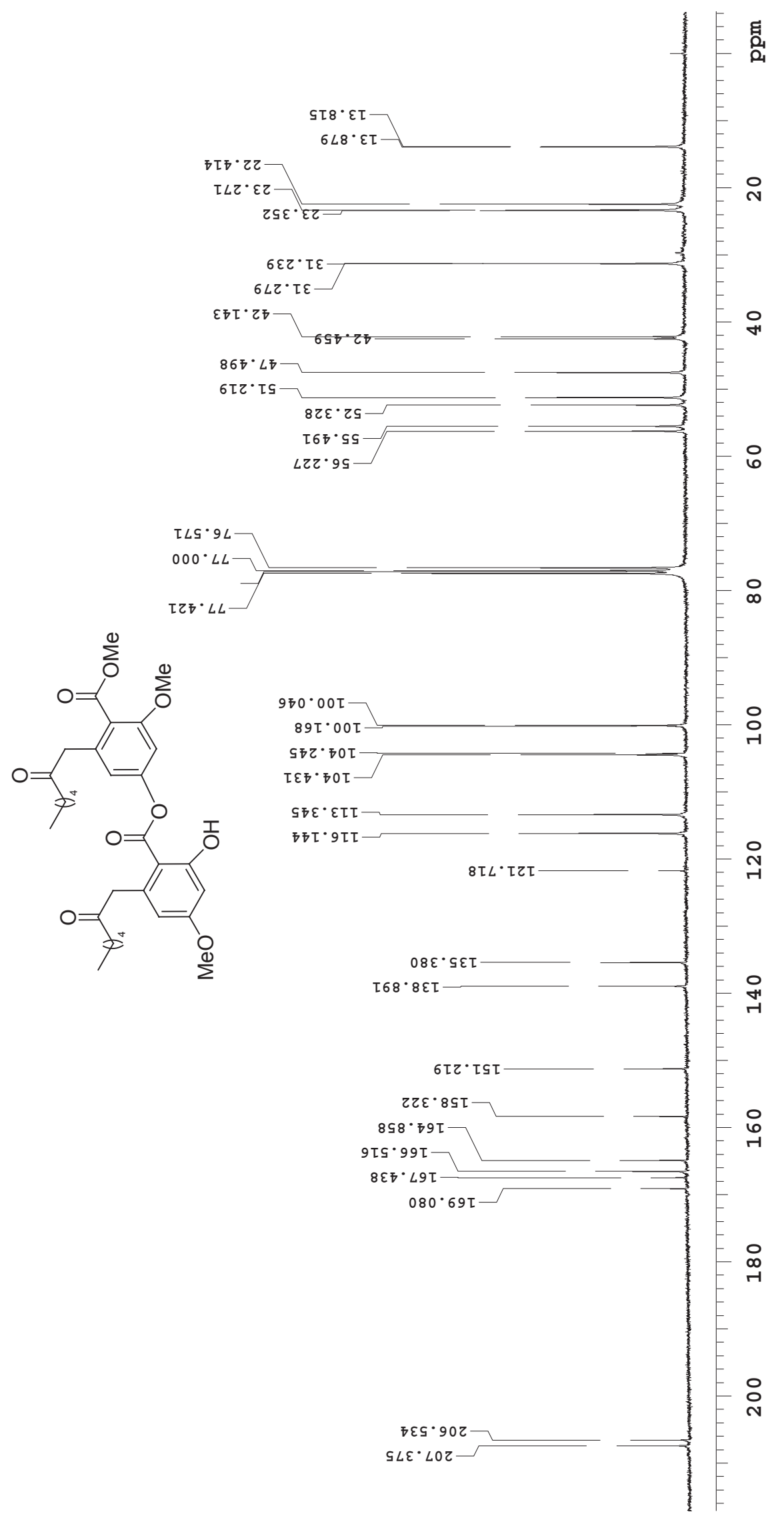

